\title{
OPEN Prevalence of cognitive impairment following chemotherapy treatment for breast cancer: a systematic review and meta-analysis
}

\begin{abstract}
Alexandra L. Whittaker ${ }^{1,2 \bowtie}$, Rebecca P. George ${ }^{1}$ \& Lucy O’Malley ${ }^{3}$
Breast cancer survival rates have markedly improved. Consequently, survivorship issues have received increased attention. One common sequel of treatment is chemotherapy-induced cognitive impairment $(\mathrm{CICl})$. $\mathrm{CICl}$ causes a range of impairments that can have a significant negative impact on quality of life. Knowledge of the prevalence of this condition is required to inform survivorship plans, and ensure adequate resource allocation and support is available for sufferers, hence a systematic review of prevalence data was performed. Medline, Scopus, CINAHL and PSYCHInfo were searched for eligible studies which included prevalence data on $\mathrm{CICl}$, as ascertained though the use of selfreport, or neuropsychological tests. Methodological quality of included studies was assessed. Findings were synthesised narratively, with meta-analyses being used to calculate pooled prevalence when impairment was assessed by neuropsychological tests. The review included 52 studies. Time-points considered ranged from the chemotherapy treatment period to greater than 10 years after treatment cessation. Summary prevalence figures (across time-points) using self-report, short cognitive screening tools and neuropsychological test batteries were $44 \%, 16 \%$ and $21-34 \%$ respectively (very low GRADE evidence). Synthesised findings demonstrate that 1 in 3 breast cancer survivors may have clinically significant cognitive impairment. Prevalence is higher when self-report based on patient experience is considered. This review highlights a number of study design issues that may have contributed to the low certainty rating of the evidence. Future studies should take a more consistent approach to the criteria used to assess impairment. Larger studies are urgently needed.
\end{abstract}

In women, breast cancer is the most frequently diagnosed cancer, with an estimated 2.3 million new cases globally per year ${ }^{1}$. Prognosis has significantly improved over time, with current 5 -year survival rates of around $90 \%$ and 10 -year survival at $80 \%{ }^{1}$. This improvement has likely been brought about largely through the ability to characterise cancer subtypes, enabling development of targeted agents, increased use of personalised medicine and consequently improved treatment success ${ }^{1}$. As a result, the number of women with breast cancer living beyond a diagnosis has grown significantly, and survivorship issues are receiving more attention, with a particular focus on quality of life issues.

Breast cancer treatment generally uses a multi-modal treatment approach utilising combinations of surgery, chemotherapy, endocrine therapy and radiotherapy, dependant on disease stage and sub-type classification ${ }^{2}$. However, chemotherapy combined with surgery, especially in advanced stages of the disease, forms the mainstay of therapy. Cognitive impairment is commonly reported in breast cancer patients both during and after cessation of treatment, and is likely triggered by multiple factors, such as endocrine therapy, the cancer itself, stress, and the hormonal changes resulting from menopause, amongst others. However, chemotherapy has been implicated as a significant contributor to this impairment. The resultant condition has been termed chemotherapy-induced cognitive impairment (CICI), cancer treatment cognitive impairment (CTCI) or cancer related cognitive impairment (CRCI) or colloquially, chemofog or chemobrain ${ }^{3,4}$. Whilst the exact mechanisms for this are unclear, proposed mechanisms include direct neurotoxic injury, a reduction in neurogenesis (new neuron formation),

\footnotetext{
${ }^{1}$ School of Animal and Veterinary Sciences, The University of Adelaide, Roseworthy Campus, Roseworthy, SA 5371, Australia. ${ }^{2}$ Faculty of Biology, Medicine and Health, University of Manchester, Manchester, UK. ${ }^{3}$ Division of Dentistry, School of Medical Sciences, Manchester Academic Health Sciences Centre, University of Manchester, Manchester, UK. ${ }^{\boxplus}$ email: alexandra.whittaker@adelaide.edu.au
} 
central nervous system white matter abnormalities, and neuroinflammation ${ }^{5}$. Most of the evidence for these theories comes from the pre-clinical literature ${ }^{5-7}$, or neuro-imaging studies ${ }^{8,9}$.

The most common neuropsychological effects that arise in CICI are impairments to visual processing, visual motor function, executive function and attention ${ }^{10}$. Sufferers typically report side effects with a wide range of severity, from subtle to more severe impairment, which may persist for up to 20 years post-chemotherapy treatment ${ }^{11}$. Impairments typically manifest as survivors feeling 'less-sharp', being unable to recall words, having episodes of metal confusion, and requiring more mental effort to perform everyday activities ${ }^{10,12}$. As a result, CICI has the potential to significantly impact patient quality of life. Furthermore, it has been suggested that a lack of prior information, coupled with minimal validation and understanding from family and health care providers leads to patients feeling disempowered, and subsequently not receiving the emotional and rehabilitative support they may need ${ }^{13}$.

The reported prevalence of CICI following treatment for breast cancer is somewhat variable. A commonly cited range from the narrative review of Janelsins et al. 2014 suggests that $12-82 \%$ of women will experience impairment as a result of their treatment regime ${ }^{14}$. This variability may be attributable to a range of patient factors such as age ${ }^{15}, \mathrm{IQ}^{16}$, menopausal status ${ }^{16}$, and education level ${ }^{17}$. Alternately, study design factors such as the employment of cross-sectional, versus longitudinal study designs, with the latter able to account for baseline pre-treatment cognitive function, likely influences rates ${ }^{18}$. Furthermore, in spite of the name, it has been shown that cognitive impairment is often present before chemotherapy treatment has commenced, potentially arising as a result of cancer itself $f^{19,20}$. This may occur via direct effects of the tumour itself, as a result of associated co-morbidities, or due to psychological factors such as worry and fatigue ${ }^{21}$. Time since treatment, also likely contributes to the variance in prevalence reported. Neuroimaging has demonstrated structural and functional changes in various patient brain regions, which likely correlate with the corresponding changes seen in executive function and memory. However, these alterations also show partial recovery over time ${ }^{22,23}$, presumably leading to an improvement in symptoms. Finally, prevalence rates may be influenced by the method used to assess cognitive impairment, with the three common methods employed being neuropsychological testing ${ }^{24}$, short cognitive screening tools ${ }^{25,26}$ and self-report ${ }^{14}$.

A preliminary database search for previous systematic reviews on prevalence of CICI was conducted. One recent systematic review was sourced which reports prevalence data for cognitive impairment in breast cancer patients ${ }^{2}$. This review is limited in scope compared to the current review since the timeline of consideration was the breast cancer treatment period, rather than longer-term follow up points. The review authors also only included longitudinal studies, which utilized objective neuropsychological tests. The earlier systematic review of Hutchinson et al. $2012^{8}$ compared objective and subjective cognitive impairments in patients with cancer but did not have a specific focus on prevalence rates. The present review differs from these previous studies by focusing specifically on CICI prevalence in patients with breast cancer as determined by self-report and objective test methods, and using meta-analytic methods and reporting guidelines designed for prevalence reviews. In the current review all study designs were eligible, not just longitudinal designs. This broad inclusion does not allow the teasing apart of the contribution of chemotherapy, as opposed to cancer itself, on impairment. However, from the point of view of the patient suffering cognitive difficulties, or health-care providers and policy makers needing evidence-based information, this distinction is of little consequence; external validity of the findings is assured. Understanding the burden of CICI will help inform survivorship strategies, and drive funding priorities for future research and targeted support, such as rehabilitation options. Furthermore, by identifying CICI incidence over the longer term this will indicate the need for longer-term support to inform cancer survivorship care plans.

Review question. The aim of this review was to synthesise the evidence on prevalence rates of cognitive impairment following chemotherapy treatment in breast cancer survivors, taking into consideration factors such as age and time since cessation of treatment.

\section{Methods}

The Joanna Briggs Institute guidelines on conducting prevalence reviews ${ }^{27}$ and the PRISMA guidelines ${ }^{28}$ were used to guide review performance and reporting. The objectives, inclusion criteria and methods of analysis for this review were specified in advance and documented in a protocol registered on PROSPERO; ref CRD42021228541.

Search strategy. The search strategy aimed to locate published studies in English. An initial search of Medline was undertaken to identify articles on the topic. Keywords used in the titles and abstracts of relevant articles, and the index terms used to describe the articles were used to develop a full search strategy for Medline via Pubmed using MeSH and free text terms. The four databases were searched in December 2020 using the developed search strategies (see supplementary material S1). Key concepts used for searching were "cancer", "chemotherapy" and "cognitive impairment". Hand searching of reference lists was performed to identify additional studies with the same selection criteria being applied. Studies published from database inception were eligible for inclusion. Publications were excluded if they were conference abstracts, review articles and grey literature.

Study selection. Following the search, all identified citations were uploaded into EndNote X8.0.1 and duplicates removed. Potentially relevant studies were retrieved in full and their citation details imported into Covidence (Veritas Health Innovation, Melbourne, Australia). Title, abstract and full text screening for assessment against the inclusion criteria for the review (see below) was performed by one reviewer (A.W), with checking of the information performed by a second reviewer (R.P.G). 
Eligibility criteria. Population. Females of any age treated with systemic chemotherapeutic agents for any form of breast cancer were considered for inclusion. Patients currently in treatment or that had ceased treatment (remission) were eligible for inclusion. Studies with patients receiving other concurrent drug therapy or with other co-morbidities were eligible for inclusion with noting (see exclusion criteria). Patients of any socioeconomic status or level of education were eligible for inclusion with noting of any differences reported. Patients that were either pre-or post-menopausal were eligible for inclusion with noting.

The following groups of patients were excluded:

- patients with previous history of traumatic brain injury, neurodegenerative conditions such as Parkinson's or Alzheimer's or severe depressive symptoms, since these conditions affect cognition either directly or indirectly ${ }^{29}$.

- patients who received palliative care (due to differences in the treatment pathways and treatment regimens) ${ }^{30}$.

- patients with metastatic CNS cancers or who have previously received CNS targeted therapy for other conditions due to the risk of direct effect on cognition as a result of the cancer/treatment ${ }^{31,32}$.

Intervention. Studies evaluating patients administered chemotherapy for breast cancer treatment were included. This included a range of common chemotherapy regimens using either sole or combination chemotherapy across any number of treatment cycles. Studies evaluating radiation and newer targeted therapies for cancer treatment were excluded. However, patients who received hormone therapy, such as tamoxifen, for prophylactic purposes following the treatment of their cancer, or accompanying local radiation therapy were eligible for inclusion with noting. This represents a deviation from the published protocol since it was discovered on screening that many studies included these patients within their chemotherapy-treatment groups. Given that these treatment strategies are commonly used, this inclusion is justified in enhancing study external validity.

Condition. Studies were included if they were investigating cognitive impairment arising as a result of interventions administered for cancer as described above. This condition may be variably described as 'chemobrain', 'chemofog', chemotherapy-induced cognitive impairment (CICI), or cancer-related cognitive impairment (CRCI). Studies where cognitive decline was indicated by any one of several commonly used modalities were eligible for inclusion. These measurement modalities included self-reported measures, imaging, or objective neuropsychological testing ${ }^{33}$.

Context and study design. Studies from any country or geographic region were eligible for inclusion with noting of location. Patients from community or clinic settings were eligible for inclusion. All study designs were eligible for inclusion. Studies that selected participants into groups based on report of cognitive decline were excluded due to the risk of sampling bias limiting the generalisability of the results.

Data extraction. Data were extracted from the included studies by one reviewer (A.W), with checking performed on all studies by a second reviewer using a modified extraction template in Covidence (supplementary material S2). The template was piloted on three studies to confirm that all required information was being considered. Key data extracted included the population sampled, chemotherapy and other treatment administered, timeframe since chemotherapy treatment or remission, identified confounding factors, method of cognitive testing and criteria for classifying impairment. Prevalence (\%) was extracted or calculated (using numerator/ denominator) from the available data. Where necessary, data were extracted from figures using GetData Graph Digitiser (version 2.26, S. Federov, Moscow, Russia). Prevalence estimates reported by sub-group, such as cognitive domain affected, age, or treatment were also recorded.

Assessment of methodological quality. Articles included in the study were assessed for methodological quality by one reviewer (AW), with confirmation provided by a second reviewer, using the JBI critical appraisal checklist and guidance notes developed for prevalence reviews (supplementary material S3) ${ }^{34}$. A study was deemed to be of high quality if it scored greater than $70 \%$, moderate quality with scores between 50 and $70 \%$ and low quality when scoring below 50\%, as described in Dijkshoorn et al. 2021 ${ }^{2}$. Summary graphs were created in Review Manager (RevMan) ([Computer program], Version 5.3. Copenhagen: The Nordic Cochrane Centre, The Cochrane Collaboration, 2014).

Data synthesis. An initial descriptive analysis of the studies was undertaken by tabulating the study characteristics and comparing against the planned sub-groups of method of cognitive assessment and timepoint following therapy cessation. Synthesis was undertaken narratively where there was heterogeneity in these study characteristics. Studies were grouped for synthesis based on time point of cognitive assessment. Basic statistical analysis was performed using Megastat Excel Add-In (version 10.3, McGraw-Hill Higher Education, New York, NY).

A meta-analysis of the prevalence rates of cognitive impairment was conducted for studies that utilised objective neuropsychological tests. Many of the included studies contained repeated observations where the unit of interest was the individual rather than observation. Consequently, these cannot be combined in a standard meta-analysis, with time-point as sub-group, since they introduce a unit-of-analysis error ${ }^{35}$. A number of approaches to perform meta-analyses in this situation have been proposed ${ }^{36}$. For this review, an All Time-points Meta-analysis (ATM) method was selected with studies at the time-points of interest being analysed separately, with a qualitative comparison being made with estimates at other time-points. Analysis was performed using the MetaXL (www.epigear.com) add-in for Microsoft Excel. A pooled prevalence figure was calculated with $95 \%$ 


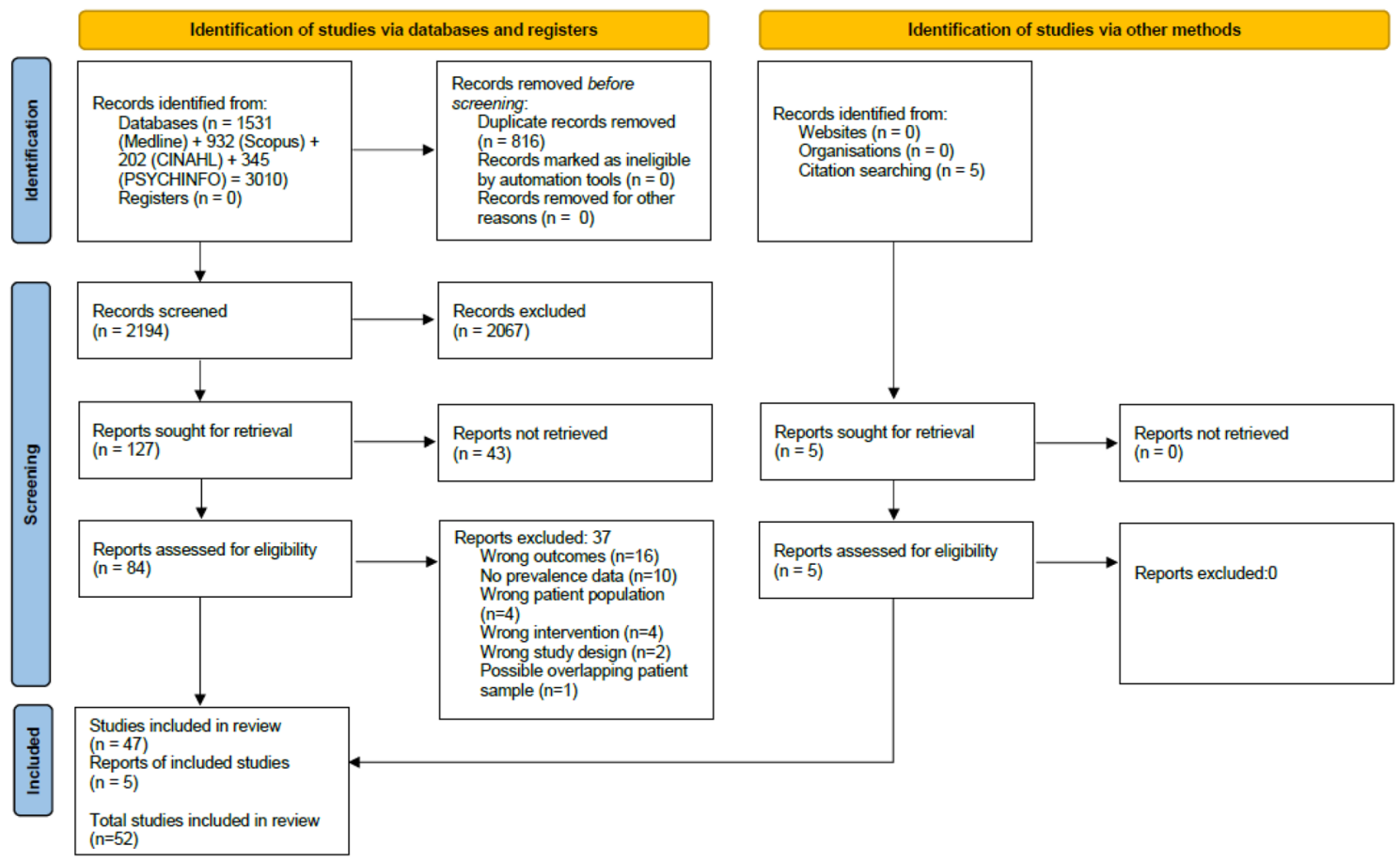

Figure 1. The PRISMA ${ }^{28}$ flow diagram for the systematic review detailing the databases searched, the number of abstracts screened and the full texts retrieved.

CI. Overall estimates were calculated with random effects models employing the DerSimonian \& Laird method as a between-study variance estimator. Cochran's Q (verifying the presence of heterogeneity) and the $I^{2}$ statistic (amount of heterogeneity) were calculated. A quality model ${ }^{37}$ was run as part of the meta-analysis where the weight of each study was calculated based on the previously calculated quality score. An $I^{2}>70 \%$ was considered substantial and these pooled data were subject to sensitivity analyses.

In a meta-analysis of prevalence, if the estimates for a study get closer to the limits of zero or one, the weight may be overestimated in the meta-analysis. Consequently, it has been recommended to transform the prevalence to a variable not constrained to the $0-1$ range with an approximately normal distribution ${ }^{38}$. The double arcsine transformation is usually preferred ${ }^{38}$ and was applied here. For final presentation, the pooled transformed proportions and corresponding CIs were back transformed.

Publication bias was examined using doi plots and the Luis Furuya-Kanamori asymmetry index (LFK index) in MetaXL. The doi plot is suggested to be more sensitive than the funnel plot, particularly when small numbers of studies are involved ${ }^{39}$.

Potential influences on prevalence estimates were investigated using subgroup analyses and meta-regression. The criteria for performing a meta-regression were derived from Cochrane guidance ${ }^{35}$. Meta-regression was performed using Comprehensive Meta-Analysis (Version 3, Borenstein, M., Hedges, L., Higgins, J., and Rothstein, H. Biostat, Englewood, NJ 2013). Age, cognitive impairment criteria stringency, sample size, methodological quality and geographical location were identified a priori as potential sources of variation in the prevalence estimates.

\section{Results}

Search results. A total of 3010 titles were identified from the electronic searches, with 5 identified from reference lists (Fig. 1). Following removal of duplicates 2194 titles remained. After title screening, 127 records remained. A further 43 records were excluded after abstract examination. Eighty-nine full-text articles were screened. Fifty-two of these met the eligibility criteria for the review, with thirty-seven being excluded at this stage (refer to supplementary material S4).

Included studies. The 52 included studies contributed 84 prevalence estimates across a series of time points in relation to chemotherapy treatment. It should be noted that the majority of studies did not seek to determine prevalence as a primary aim of the study, with the data being presented as one of a number of outcomes reported. Three studies did however have a focus on prevalence as expounded in their study aims ${ }^{40-42}$. The majority of studies adopted an observational study design (48 studies, 92\%). The remainder were randomised controlled trials (RCT). The studies that employed RCT designs were evaluations where the primary study question related to differences between different chemotherapy regimes or alternate therapeutic strategies and there was no selection into groups based on presence of cognitive impairment (an exclusion criterion for this review). Out of the studies that were observational in nature, 25 (52\%) were cohort designs, 20 (42\%) cross-sectional and 13 (27\%) prospective longitudinal. Sample sizes were generally small with a range from 20 to 1147. Studies with larger sample sizes tended to employ self-report measures rather than objective test modalities. 
With the exception of two studies ${ }^{43,44}$, data came from countries with high-income economies (World Bank Classification). The vast majority of study data originated from the continents of North America and Europe (42\% and $44 \%$ respectively). Age of study participants was generally similar across studies and to be expected given the breast cancer age risk. The pooled mean across the studies was age 52 (range of included study means: 45-71). Three studies ${ }^{15,45,46}$ were specifically interested in older patients and included only women over the age of 65 .

Consistent with clinical practice, women received a range of chemotherapy drugs as part of their treatment cycles across the studies. Common combinations reported were FEC (5-fluorouracil, epirubicin, and cyclophosphamide), CMF (cyclophosphamide, methotrexate and 5-fluorouracil), doxorubicin + paclitaxel, doxorubicin + cyclophosphamide. This information has not been presented in the review due to a general lack of separation of outcome data based on treatment in the primary studies, necessitating assimilation as a group. In most studies $(n=43)$, a significant percentage of patients, especially at later follow-up points were receiving hormonal therapy such as tamoxifen. In the remaining studies, patients on hormonal therapies were either excluded from study design or studies were conducted early in the treatment phase (see Tables 1, 2 and 3). Hormonal therapies have been shown to influence cognition ${ }^{47,48}$. These agents are administered frequently and can be considered a standard part of treatment. Inclusion of these data hence supports the external validity of the review's findings.

The included studies generally assessed cognition at similar time-points to coincide with typical phases of breast cancer treatment. Due to this similarity studies were grouped based on these time points for further assimilation, labelled T1-T7 (refer to supplementary material S5). Data from baseline, pre-chemotherapy assessments (T0), were not extracted as part of this review since we did not have as an inclusion criterion that studies had to be longitudinal. Further, the review question relates to prevalence of impairment after breast cancer treatment, not any measured decline as a result of treatment.

The included studies utilized a range of methods for assessment of cognitive impairment. It was considered that these differences could introduce substantial heterogeneity when assimilating prevalence estimates. Hence, assimilation was undertaken based on use of three different methods; self-report measures, short cognitive screening tools, and objective neuropsychological test batteries (supplementary material S6). Fifteen studies utilized self-report measures, nine employed short cognitive screening tools and a majority of 35 used neuropsychological test batteries. Seven studies utilized two of these testing modalities ${ }^{15,42,49-53}$. Studies using objective cognitive tests for the most part used tests which spanned the various cognitive domains of executive function, language function, motor, processing speed, verbal and visual learning and memory, visuo-spatial function and working memory. From here on, review data is presented based on these 3 groupings of outcome assessment method (refer to Table 1, 2, and 3).

Methodological quality. A summary of the methodological quality assessment for the included articles is provided in Fig. 2 and supplementary material S7. Thirty-five (67\%) studies were considered to be of high quality, fifteen (29\%) of moderate quality and only two ${ }^{43,44}(4 \%)$ studies of low quality (see Tables 1, 2 and 3 ). The main domains of concern were participant sampling, sample size, condition measurement and statistical analysis. Of these, the outcomes of assessment of risk in the domains of sample size and condition measurement are considered to have the most impact on the review outcomes. However, use of a quality model in meta-analysis and evaluating the impact of sample size by meta-regression minimizes this impact.

Prevalence estimates based on self-report measures of cognitive impairment. It was considered that there was significant variation in the studies that used self-report measures in terms of method of assessment of cognitive impairment, timing of assessment in relation to chemotherapy treatment and form of report (based on cognitive domain rather than overall impairment). Therefore, these results are presented narratively.

Fifteen studies (Table 1) examined prevalence of cognitive impairment after breast cancer treatment contributing 22 prevalence estimates across all study time-points with the exception of T6. There were a number of studies with large sample sizes in this group ${ }^{54,56,58}$, however the median sample size for the group remained modest at 85 (range 31-1147). Studies utilized a variety of methods to assess impairment which ranged from validated selfreport instruments such as FACT-Cog or PAOFI, to semi-structured interviews or Likert responses to questions on cognitive function. Seven (47\%) of studies used the former. When the studies employing validated tests were contrasted with those using non-validated methods median global impairment across the time points was $46 \%$ and $39.5 \%$ respectively, with no significant difference in prevalence rate between the two subgroups (two tailed T-test; $t(6,5)=-1.2, p=0.27)$.

Prevalence rates for global impairment varied from 21 to $83 \%$ (mean $44 \%$ ) across all time-points. Noting the paucity of data at T6 and T7, no obvious downward trend in impairment across time is evident (Fig. 3). Two studies in this sub-group focused on CICI in older women over the age of $65^{15,45}$ reporting prevalence rates of $51 \%{ }^{45}$, and $34 \%{ }^{15}$. Whilst, the former is clearly above the mean reported above of $44 \%$, the value within Lange et al. $2016^{15}$ actually falls under the calculated average.

Prevalence estimates based on use of short cognitive screening tools to measure cognitive impairment. There was considerably less variation in assessment method for cognitive impairment, and time points of measure for the studies that utilised short cognitive screening measures compared to those employing self-report. However, at each time point there were fewer than five studies. Study has suggested that at least 5 studies are needed to achieve statistical power from random effects meta-analyses that are superior to the individual contributing studies $^{90}$. Studies within this group were therefore not subjected to meta-analytic techniques and are reported narratively.

Nine studies (Table 2) utilized short cognitive screening tools as part of this grouping, contributing 17 prevalence estimates. No studies performed longer-term follow up at the T6 and T7 time-points. Sample size ranged 


\begin{tabular}{|c|c|c|c|c|c|c|c|c|c|c|}
\hline Study & Study design & Location & $\begin{array}{l}\text { Endocrine } \\
\text { therapy use }\end{array}$ & Sample size & $\begin{array}{l}\text { Sample age } \\
\text { (range, mean) }\end{array}$ & $\begin{array}{l}\text { Assessment } \\
\text { method }\end{array}$ & $\begin{array}{l}\text { Methodological } \\
\text { quality }\end{array}$ & $\begin{array}{l}\text { Definition } \\
\text { of cognitive } \\
\text { decline }\end{array}$ & Time points & $\begin{array}{l}\text { Prevalence } \\
\%(95 \% \\
\text { CIs where } \\
\text { available })\end{array}$ \\
\hline $\begin{array}{l}\text { Buchanan } \\
2015^{54}\end{array}$ & $\begin{array}{l}\text { Cross-sec- } \\
\text { tional }\end{array}$ & United States & Yes & $\begin{array}{l}\text { Chemother- } \\
\text { apy }=288 \\
\text { Chemo- } \\
\text { therapy+ Hor- } \\
\text { mone }=859\end{array}$ & $34-82$ & FACT-Cog & High & $\begin{array}{l}\text { Reponses } \\
\text { of cognitive } \\
\text { complaints } \\
\text { "sometimes", } \\
\text { "often", or } \\
\text { "always" }\end{array}$ & Mixed $^{*}$ & $\begin{array}{l}74 \text { Chemo- } \\
\text { therapy } \\
78 \text { Chemo- } \\
\text { therapy+ } \\
\text { Hormone }\end{array}$ \\
\hline Debess $2010^{50}$ & Cohort & Denmark & Yes & 75 & $29-59,47.2$ & $\begin{array}{l}\text { Author- } \\
\text { derived ques- } \\
\text { tions assessed } \\
\text { on 7-point } \\
\text { scale }\end{array}$ & High & Scores of 5-7 & T2 & $\begin{array}{l}\text { Memory: } 29 \\
\text { Concentra- } \\
\text { tion: } 13 \\
\text { Mental } \\
\text { fatigue: } 33 \\
\text { Vigour: } 43\end{array}$ \\
\hline Ganz $2013^{55}$ & Cohort & United States & Yes & 189 & 51.8 & PAOFI & Moderate & \begin{tabular}{|l} 
Classified \\
based on \\
PAOFI score \\
as high \\
range versus \\
normal/no \\
complaints \\
\end{tabular} & Mixed $^{*}$ & $\begin{array}{l}\text { Memory: } 23 \\
\text { High level } \\
\text { cognition: } 19\end{array}$ \\
\hline Hurria $2006^{45}$ & $\begin{array}{l}\text { Prospective } \\
\text { Longitudinal }\end{array}$ & United States & Yes & 45 & $65-84,70$ & Squire & Moderate & $\begin{array}{l}\text { Unclear but } \\
\text { based on score }\end{array}$ & T3 & 51 \\
\hline $\begin{array}{l}\text { Janelsins } \\
2017^{56}\end{array}$ & $\begin{array}{l}\text { Prospective } \\
\text { Longitudinal }\end{array}$ & United States & Yes & 505 & $22-81,53.4$ & FACT-Cog & High & \begin{tabular}{|l|} 
A $1 / 2$ standard \\
deviation \\
representing \\
a minimal \\
clinically \\
important \\
difference
\end{tabular} & $\mathrm{T} 2, \mathrm{~T} 3$ & $\begin{array}{l}\text { T2: } 45 \\
\text { T3: } 37\end{array}$ \\
\hline Jenkins $2006^{51}$ & Cohort & UK & Yes & 85 & 51.5 & Broadbent & High & $\begin{array}{l}\text { Self-report of } \\
\text { change }\end{array}$ & $\mathrm{T} 2, \mathrm{~T} 3$ & $\begin{array}{l}\text { T2: } \\
\text { Memory: } 83 \\
\text { Concentra- } \\
\text { tion: } 80 \\
\text { T3: } \\
\text { Memory: } 60 \\
\text { concentra- } \\
\text { tion: } 45\end{array}$ \\
\hline $\begin{array}{l}\text { Koppelmans } \\
2012^{11}\end{array}$ & Cohort & Netherlands & No & 196 & $50-80,64.1$ & $\begin{array}{l}\text { Basic self- } \\
\text { report }\end{array}$ & High & $\begin{array}{l}\text { Yes response } \\
\text { to question }\end{array}$ & T7 & \begin{tabular}{|l|} 
More prob- \\
lems remem- \\
bering: 53 \\
Forgetting \\
(daily) pur- \\
suits: 43 \\
Word-finding \\
problems: 38
\end{tabular} \\
\hline Lange $2016^{15}$ & Cohort & France & Yes & 58 & $65-81,70$ & FACT-Cog & High & $\begin{array}{l}\text { A difference } \\
\text { of more than } \\
10 \% \text { between } \\
\text { baseline } \\
\text { and T2 was } \\
\text { considered } \\
\text { clinically } \\
\text { significant }\end{array}$ & T2 & \begin{tabular}{|l|} 
Perceived \\
Cognitive \\
Impairment: \\
34 \\
Perceived \\
Cognitive \\
abilities: 49 \\
(80 over \\
75 years)
\end{tabular} \\
\hline Ng $2018^{52}$ & $\begin{array}{l}\text { Prospective } \\
\text { Longitudinal }\end{array}$ & Singapore & Yes & 166 & 50.7 & FACT-Cog & High & $\begin{array}{l}\text { A drop of } 10.6 \\
\text { points in the } \\
\text { global score } \\
\text { from baseline }\end{array}$ & $\mathrm{T} 1, \mathrm{~T} 3$ & $\begin{array}{l}\text { T1: } 22 \\
\text { T3: } 31\end{array}$ \\
\hline Rey $2012^{57}$ & Cohort & France & Yes & 222 & $18-40,37$ & $\begin{array}{l}\text { Telephone } \\
\text { Interview }\end{array}$ & High & $\begin{array}{l}\text { Women who } \\
\text { reported } \\
\text { memory } \\
\text { andor atten- } \\
\text { tion troubles } \\
\text { "very often" or } \\
\text { "often" }\end{array}$ & T3, T4, T5 & $\begin{array}{l}\text { T3:37 } \\
\text { T4: } 37 \\
\text { T5: } 42\end{array}$ \\
\hline $\begin{array}{l}\text { Schagen } \\
1999^{53}\end{array}$ & Cohort & Netherlands & Yes & 39 & 47.1 & $\begin{array}{l}\text { Interview } \\
\text { with questions } \\
\text { scored on a } \\
\text { Likert scale }\end{array}$ & Moderate & $\begin{array}{l}\text { A score of } 2 \\
\text { (moderate) } \\
\text { or more was } \\
\text { considered a } \\
\text { distinct com- } \\
\text { plaint about } \\
\text { the cognitive } \\
\text { functioning } \\
\text { in the domain } \\
\text { concerned }\end{array}$ & T5 & $\begin{array}{l}\text { Concentra- } \\
\text { tion prob- } \\
\text { lems: } 31 \\
\text { Memory } \\
\text { problems: } 21 \\
\text { Thinking: } 8 \\
\text { Language: } 8\end{array}$ \\
\hline
\end{tabular}




\begin{tabular}{|c|c|c|c|c|c|c|c|c|c|c|}
\hline Study & Study design & Location & $\begin{array}{l}\text { Endocrine } \\
\text { therapy use }\end{array}$ & Sample size & $\begin{array}{l}\text { Sample age } \\
\text { (range, mean) }\end{array}$ & $\begin{array}{l}\text { Assessment } \\
\text { method }\end{array}$ & $\begin{array}{l}\text { Methodological } \\
\text { quality }\end{array}$ & $\begin{array}{l}\text { Definition } \\
\text { of cognitive } \\
\text { decline }\end{array}$ & Time points & $\begin{array}{l}\text { Prevalence } \\
\%(95 \% \\
\text { CIs where } \\
\text { available) }\end{array}$ \\
\hline $\begin{array}{l}\text { Schmidt } \\
2016^{58}\end{array}$ & $\begin{array}{l}\text { Cross-sec- } \\
\text { tional }\end{array}$ & United States & Yes & 904 & $\begin{array}{l}48.8 \text { (across } \\
\text { range of can- } \\
\text { cer types) }\end{array}$ & \begin{tabular}{|l} 
Based on \\
Quality of Life \\
in Adult Can- \\
cer Survivors \\
(QLACS) with \\
some amend- \\
ment
\end{tabular} & High & $\begin{array}{l}\text { An endorse- } \\
\text { ment of the } \\
\text { items was } \\
\text { categorised } \\
\text { as perceived } \\
\text { cognitive } \\
\text { decline }\end{array}$ & Unclear & 58 \\
\hline Shilling $2007^{59}$ & Cohort & UK & No & 85 & 51.7 & Interview & Moderate & $\begin{array}{l}\text { An endorse- } \\
\text { ment of the } \\
\text { items }\end{array}$ & $\mathrm{T} 2, \mathrm{~T} 4$ & $\begin{array}{l}\text { Memory } \\
\text { T2: } 83 \\
\text { T4: } 60 \\
\text { Concentra- } \\
\text { tion: } \\
\text { T2: } 78 \\
\text { T3: } 45\end{array}$ \\
\hline Tager $2010^{60}$ & Cohort & United States & No & 31 & $\begin{array}{l}46.95-70.96 \\
60.7\end{array}$ & $\begin{array}{l}\text { Participants } \\
\text { asked to rate } \\
\text { perceived } \\
\text { memory } \\
\text { abilities on } \\
\text { a five-point } \\
\text { Likert scale }\end{array}$ & Moderate & $\begin{array}{l}\text { Ratings }>2 \\
\text { were coded } \\
\text { as cognitive } \\
\text { problems }\end{array}$ & T2,T3 & $\begin{array}{l}\text { T2: } 43 \\
\text { T3: } 46\end{array}$ \\
\hline $\begin{array}{l}\text { Van Dam } \\
1998^{42}\end{array}$ & RCT & Netherlands & Yes & $\begin{array}{l}\text { FEC }^{\mp}: 36 \\
\text { CTC }^{\neq}: 34\end{array}$ & $\begin{array}{l}\text { FEC }^{\mp}: 48.1 \\
\text { CTC }^{\mp}: 45.5\end{array}$ & $\begin{array}{l}\text { Cognitive } \\
\text { problems } \\
\text { in daily life } \\
\text { interview }\end{array}$ & High & $\begin{array}{l}\text { An endorse- } \\
\text { ment of the } \\
\text { items was } \\
\text { categorised } \\
\text { as perceived } \\
\text { cognitive } \\
\text { decline }\end{array}$ & T5 & \begin{tabular}{|l|} 
Concentra- \\
tion: high- \\
dose:38 \\
Standard- \\
dose: 31 \\
Memory \\
High-dose: 32 \\
Standard- \\
dose: 28 \\
Thinking: \\
High-dose: 21 \\
Standard- \\
dose: 11 \\
Language: \\
High-dose:12 \\
Standard- \\
dose: 11
\end{tabular} \\
\hline
\end{tabular}

Table 1. Summary of included studies that used self-report measures of cognitive impairment. *Time-points measured in study cross time-points used in this review preventing accurate assimilation. $\neq \mathrm{T} 1=$ During chemotherapy treatment, T2 = Just after cessation of treatment, T3 $=6$ months after treatment cessation, $\mathrm{T} 4=1$ year after treatment cessation, $\mathrm{T} 5=2-3$ years after treatment cessation, $\mathrm{T} 6=5-10$ years after treatment cessation, $\mathrm{T} 7=\geq 10$ years after treatment cessation. TFluorouracil, epirubicin, cyclophosphamide (standard dose). ${ }^{\neq}$Four cycles of FEC followed by cyclophosphamide, thiotepa, and carboplatin (high dose).

from 20 to 475 (mean =170). Five different validated methods of assessing impairment were employed (Table 1). With the exception of the Doors and People test these methods assess a range of cognitive domains expected to be impacted by chemotherapy administration. In two studies ${ }^{25,26}$ test outcomes were reported based on a banding of scores as mild, moderate or severe. For the purposes of assimilation, only prevalence estimates for moderate-severe impairment have been considered.

In contrast to studies that used self-report none of these studies specifically examined older populations. However, three of these studies ${ }^{25,43,62}$ had a wider age range with patients above 65 years of age being included. This has not however led to a substantially increased mean participant age in comparison to the other studies.

Prevalence rates across all time-points ranged from 0 to 50 . The mean prevalence was $16 \%$. Exclusion of the four prevalence estimates of zero provided by Pilai et al. $2019^{43}$ leads to a range of $4 \%-50 \%$, with mean prevalence of $21 \%$. This action may be justified given the low quality rating assigned to this study. There was a general downward trend in prevalence across time, but range at each time-point (with the exception of T3) was large (Fig. 4).

Prevalence estimates based on use of objective neuropsychological testing to measure cognitive impairment. Thirtyfive studies (Table 3) utilized objective neuropsychological tests to screen for cognitive impairment. These studies contributed 46 prevalence estimates. Sample size ranged from 20 to 136 (median=53) having the lowest range and average of all the groups. Studies reported prevalence across all time points of interest, although only one estimate was reported at each of the longer-term follow up points (T6 and T7). Two studies in the group evaluated women over the age of $65^{15,46}$. The neuropsychological tests used were all established, validated tests which when administered in a battery broadly encompass the major cognitive domains. Two studies only employed one test for cognitive assessment ${ }^{44,64}$, and as such did not evaluate all cognitive domains.

There were differences between the studies in the criteria used to determine cognitive impairment. In general a diagnosis of impairment was made based on two factors being met: (1) the level of decline in score relative to some reference value, for example healthy control patients (2) that this decline occurred in a pre-determined number of tests. Fourteen (40\%) of the studies used 2 standard deviation declines to guide level but there was 


\begin{tabular}{|c|c|c|c|c|c|c|c|c|c|c|}
\hline Study & Study design & Location & $\begin{array}{l}\text { Endocrine } \\
\text { therapy use }\end{array}$ & Sample size & $\begin{array}{l}\text { Sample } \\
\text { age (range, } \\
\text { mean) }\end{array}$ & $\begin{array}{l}\text { Assessment } \\
\text { method }\end{array}$ & $\begin{array}{l}\text { Methodological } \\
\text { quality }\end{array}$ & $\begin{array}{l}\text { Definition } \\
\text { of cognitive } \\
\text { decline }\end{array}$ & $\begin{array}{l}\text { Time point(s) } \\
\neq\end{array}$ & $\begin{array}{l}\text { Prevalence \% } \\
\text { (95\% CIs where } \\
\text { available) }\end{array}$ \\
\hline Biglia $2012^{49}$ & $\begin{array}{l}\text { Prospective } \\
\text { Longitudinal }\end{array}$ & Italy & No & 40 & $38-65,51$ & MMSE & Moderate & $\begin{array}{l}\text { Score under } \\
\text { the mean }\end{array}$ & $\mathrm{T} 2$ & 31 \\
\hline $\begin{array}{l}\text { Brezden } \\
2000^{25}\end{array}$ & Cohort & Canada & Yes & $\begin{array}{l}71 \text { across two } \\
\text { groups: } \\
\text { (1) Currently } \\
\text { receiving } \\
\text { chemother- } \\
\text { apy, and (2) } \\
\text { one year after } \\
\text { cessation }\end{array}$ & $\begin{array}{l}34-70,49 \\
26-61,46\end{array}$ & HSCS & High & $\begin{array}{l}\text { Classifica- } \\
\text { tion as mild, } \\
\text { moderate or } \\
\text { severe accord- } \\
\text { ing to pattern } \\
\text { of scores as } \\
\text { described by } \\
\text { test authors }\end{array}$ & $\mathrm{T} 1, \mathrm{~T} 4$ & \begin{tabular}{|l} 
T1 \\
Mild: 13 \\
Moderate: 10 \\
Severe: $12 / 31=39$ \\
T4 \\
Mild: 20 \\
Moderate: 28 \\
Severe: $9 / 40=23$
\end{tabular} \\
\hline Fan $2005^{26}$ & Cohort & Canada & Yes & 81 & 48 (median) & HSCS & High & $\begin{array}{l}\text { Scores from } \\
\text { each item on } \\
\text { the test were } \\
\text { subject to an } \\
\text { algorithm } \\
\text { which gener- } \\
\text { ated bands of } \\
\text { impairment }\end{array}$ & $\mathrm{T} 2, \mathrm{~T} 4, \mathrm{~T} 5$ & \begin{tabular}{|l|} 
T2 \\
Mild: 35 \\
Mod-Severe: 16 \\
T4 \\
Mild: 30 \\
Mod-severe: 4 \\
T5: \\
Mild: 21 \\
Mod-severe: 4 \\
\end{tabular} \\
\hline Fontes $2016^{40}$ & $\begin{array}{l}\text { Prospective } \\
\text { longitudinal }\end{array}$ & Portugal & Yes & 475 & 54.7 (median) & MoCA & High & $\begin{array}{l}\text { A MoCA } \\
\text { score at least } \\
2.0 \text { standard } \\
\text { deviations } \\
\text { below age- } \\
\text { and educa- } \\
\text { tion-adjusted } \\
\text { cut-offs }\end{array}$ & T3, T5 & $\begin{array}{l}\text { T3: } 7 \\
\text { T5: } 8\end{array}$ \\
\hline $\mathrm{Ng} 2018^{52}$ & $\begin{array}{l}\text { Prospective } \\
\text { Longitudinal }\end{array}$ & Singapore & Yes & 166 & 50.7 & $\begin{array}{l}\text { Headminder } \\
\text { computerized } \\
\text { test }\end{array}$ & High & \begin{tabular}{|l|} 
Reliable \\
change index \\
(RCI) score \\
calculated to \\
determine \\
cognitive \\
decline in \\
each cognitive \\
domain. A \\
RCI score of \\
lower than \\
-1.5 was used \\
as criteria for \\
decline
\end{tabular} & $\mathrm{T} 1, \mathrm{~T} 3$ & $\begin{array}{l}\text { T1: } 6.1-21.6 \\
\text { T3: } 1.2-14.6\end{array}$ \\
\hline Pillai $2019^{43}$ & $\begin{array}{l}\text { Prospective } \\
\text { longitudinal }\end{array}$ & India & Yes & 152 & $27-72,47$ & MMSE & Low & $\begin{array}{l}\text { MMSE score } \\
\text { of } \leq 24 \text { (out } \\
\text { of } 30)\end{array}$ & $\mathrm{T} 2, \mathrm{~T} 3, \mathrm{~T} 4, \mathrm{~T} 5$ & $\begin{array}{l}\text { T2: } 0 \\
\text { T3: } 0 \\
\text { T4: } 0 \\
\text { T5: } 0 \\
\end{array}$ \\
\hline $\begin{array}{l}\text { Prokasheva } \\
2011^{61}\end{array}$ & Cohort & Israel & Yes & 20 & $30-57,49.3$ & $\begin{array}{l}\text { Doors and } \\
\text { people test }\end{array}$ & Moderate & $\begin{array}{l}\text { Below } 1 \text { SD as } \\
\text { indicative of } \\
\text { mild impair- } \\
\text { ment and } \\
\text { below 2SD } \\
\text { for moderate } \\
\text { impairment }\end{array}$ & T5 & 40 \\
\hline $\begin{array}{l}\text { Ramalho } \\
2017^{62}\end{array}$ & Cohort & Portugal & Yes & 418 & $27-87$ & MoCA & High & $\begin{array}{l}\text { MoCA score } \\
\text { values at least } \\
1.5 \text { standard } \\
\text { deviations } \\
\text { below age- } \\
\text { and educa- } \\
\text { tion-adjusted } \\
\text { cut-offs } \\
\end{array}$ & T3 & $8(5.8,11)$ \\
\hline Tchen $2003^{63}$ & Cohort & Canada & No & 110 & $27-60,48$ & HSCS & High & $\begin{array}{l}\text { Based on } \\
\text { standard test } \\
\text { criteria }\end{array}$ & T1 & \begin{tabular}{|l|}
$\mathrm{T} 1$ \\
Mild: 34 \\
Moderate: 2 \\
Severe: 14
\end{tabular} \\
\hline
\end{tabular}

Table 2. Summary of included studies employing short cognitive screening tools to assess cognitive impairment. $\neq \mathrm{T} 1$ = During chemotherapy treatment, $\mathrm{T} 2=$ Just after cessation of treatment, $\mathrm{T} 3=6$ months after treatment cessation, $\mathrm{T} 4=1$ year after treatment cessation, $\mathrm{T} 5=2-3$ years after treatment cessation.

heterogeneity even between these in the number of tests with declining scores required to satisfy the diagnostic criteria (see Table 3). Studies that considered a one SD decline as criteria for impairment, or were not clear on the level used, were considered less stringent (see meta-analysis). Three studies ${ }^{72,78,79}$ reported test outcomes by cognitive domain or individual test, rather than global impairment rendering these data harder to assimilate with the other studies. 


\begin{tabular}{|c|c|c|c|c|c|c|c|c|c|c|}
\hline Study & Study design & Location & $\begin{array}{l}\text { Endocrine } \\
\text { therapy use }\end{array}$ & Sample size & \begin{tabular}{|l|} 
Sample age \\
(range, mean)
\end{tabular} & $\begin{array}{l}\text { Number } \\
\text { of tests in } \\
\text { battery }\end{array}$ & $\begin{array}{l}\text { Methodological } \\
\text { quality }\end{array}$ & $\begin{array}{l}\text { Definition } \\
\text { of cognitive } \\
\text { decline }\end{array}$ & $\begin{array}{l}\text { Time point }(\mathbf{s}) \\
\neq\end{array}$ & $\begin{array}{l}\text { Prevalence } \\
\% \text { (95\% } \\
\text { CIs where } \\
\text { available) }\end{array}$ \\
\hline $\begin{array}{l}\text { Andryszak } \\
2018^{64}\end{array}$ & Cohort & Poland & No & 31 & 52.4 & 1 & Moderate & $\begin{array}{l}\text { Scores lower } \\
\text { than } 2 \text { SD } \\
\text { compared to } \\
\text { control group } \\
\text { averages }\end{array}$ & $\mathrm{T} 1, \mathrm{~T} 2$ & $\begin{array}{l}\text { T1:32 } \\
\text { T2: } 26\end{array}$ \\
\hline Biglia $2012^{49}$ & $\begin{array}{l}\text { Prospective } \\
\text { Longitudinal }\end{array}$ & Italy & No & 40 & $38-65,51$ & 10 & Moderate & $\begin{array}{l}\text { "Unsatisfying } \\
\text { score" not } \\
\text { defined in } \\
\text { report }\end{array}$ & $\mathrm{T} 2$ & 15 \\
\hline Collins $2009^{65}$ & Cohort & Canada & Yes & 53 & $50-65,57.9$ & 18 & High & $\begin{array}{l}\mathrm{SRB}^{*} \text { scores } \\
\text { of }-2 \text { or less } \\
\text { on at least two } \\
\text { tests }\end{array}$ & $\mathrm{T} 2, \mathrm{~T} 4$ & $\begin{array}{l}\text { T2: } 34 \\
\text { T4: } 11\end{array}$ \\
\hline Collins $2013^{66}$ & $\begin{array}{l}\text { Prospective } \\
\text { longitudinal }\end{array}$ & Canada & No & 60 & 52.4 & 17 & High & $\begin{array}{l}\text { Scores lower } \\
\text { than } 2 \text { SD on } \\
\text { at least two } \\
\text { tests }\end{array}$ & $\begin{array}{l}\mathrm{T} 1 \text { (measured } \\
\text { at end of each } \\
\text { cycle) }\end{array}$ & $\begin{array}{l}30 \text { (mean } \\
\text { across all } \\
\text { cycles) }\end{array}$ \\
\hline Collins $2014^{67}$ & Cohort & Canada & Yes & 56 & 51.8 & 19 & High & $\begin{array}{l}\text { SRB scores of } \\
-2 \text { or less on } \\
\text { at least two } \\
\text { tests }\end{array}$ & $\begin{array}{l}\text { T1 (measured } \\
\text { at end of each } \\
\text { cycle) } \\
\text { T4 }\end{array}$ & $\begin{array}{l}\text { T1: } 48 \\
\text { T4: } 22\end{array}$ \\
\hline Debess $2010^{50}$ & Cohort & Denmark & Yes & 75 & $29-59,47.2$ & 4 & High & $\begin{array}{l}\text { Significant } \\
\text { changes } \\
\text { (between } \\
5 \text { and 95th } \\
\text { percentile in } \\
\text { controls) on at } \\
\text { least two tests }\end{array}$ & $\mathrm{T} 2$ & 4 \\
\hline $\begin{array}{l}\text { Hermelink } \\
2007^{68}\end{array}$ & RCT & Germany & Yes & 101 & 48.6 & 12 & High & $\begin{array}{l}\text { Reliable- } \\
\text { change index } \\
\text { (RCI) with a } \\
\text { probability } \\
\text { of error set at } \\
10 \%\end{array}$ & $\mathrm{~T} 1$ & 27 \\
\hline $\begin{array}{l}\text { Hermelink } \\
2017^{69}\end{array}$ & Cohort & Germany & Yes & 91 & $\begin{array}{l}27.3-64.9 \\
52.3\end{array}$ & 18 & High & $\begin{array}{l}\text { Five or more } \\
\text { scores below } \\
1.5 \text { standard } \\
\text { deviations } \\
\text { and/or four } \\
\text { below } 2 \text { stand- } \\
\text { ard deviations }\end{array}$ & $\mathrm{T} 2, \mathrm{~T} 4$ & $\begin{array}{l}\text { T2: } 6 \\
\text { T4: } 18\end{array}$ \\
\hline Hurria $2006^{46}$ & $\begin{array}{l}\text { Prospective } \\
\text { longitudinal }\end{array}$ & United States & Yes & 28 & $65-84,71$ & 13 & Moderate & $\begin{array}{l}\text { Scores lower } \\
\text { than } 2 \text { SD on } \\
\text { at least two } \\
\text { tests }\end{array}$ & $\mathrm{T} 3$ & 39 \\
\hline Jansen $2011^{70}$ & $\begin{array}{l}\text { Prospective } \\
\text { longitudinal }\end{array}$ & United States & Yes & 71 & $30-65,50.3$ & 4 & High & $\begin{array}{l}\text { Scores lower } \\
\text { than } 1 \text { SD on } \\
\text { at least two } \\
\text { tests }\end{array}$ & $\mathrm{T} 1, \mathrm{~T} 2, \mathrm{~T} 3$ & $\begin{array}{l}\text { T1:23 } \\
\text { T2:52 } \\
\text { T3:20 }\end{array}$ \\
\hline Jenkins $2006^{51}$ & Cohort & UK & Yes & 85 & 51.5 & 7 & High & $\begin{array}{l}\text { Reliable } \\
\text { change index } \\
\text { on at least two } \\
\text { measurement }\end{array}$ & $\mathrm{T} 2, \mathrm{~T} 3$ & $\begin{array}{l}\text { T2: } 20 \\
\text { T3: } 18\end{array}$ \\
\hline $\operatorname{Jim} 2009^{71}$ & Cohort & United States & Yes & 97 & 50 & 9 & High & $\begin{array}{l}\text { Scores lower } \\
\text { than } 1.5 \mathrm{SD} \\
\text { on at least two } \\
\text { tests }\end{array}$ & T3 & 34 \\
\hline Jung $2014^{72}$ & $\begin{array}{l}\text { Cross sec- } \\
\text { tional study }\end{array}$ & Korea & Yes & 32 & $31-61,46$ & 3 & High & $\begin{array}{l}\text { Cutoff as } 5 / 4 \\
\text { on digit span- } \\
\text { DS F; } 4 / 3 \text { on } \\
\text { digit span B; } \\
28-30 / 25 \text { on } \\
\text { Controlled } \\
\text { Oral Word } \\
\text { Association- } \\
\text { COWA) }\end{array}$ & $\mathrm{T} 2$ & $\begin{array}{l}\text { DSF } \\
\text { Mild: } 32 \\
\text { Moderate: } 13 \\
\text { DSB } \\
\text { Mild: } 32 \\
\text { Moderate: } 42 \\
\text { COWA }{ }^{\star}(\mathrm{A}) \\
\text { Mild: } 3 \\
\text { Moderate: } 58 \\
\text { COWA (B) } \\
\text { Mild: } 52\end{array}$ \\
\hline Kesler $2017^{73}$ & Cohort & United States & Yes & 31 & $34-65,48.6$ & 4 & Moderate & $\begin{array}{l}\text { Scores lower } \\
\text { than } 1.5 \mathrm{SD} \\
\text { on at least two } \\
\text { tests or lower } \\
\text { than } 2 \mathrm{SD} \text { on } \\
\text { any one test }\end{array}$ & T4 & 55 \\
\hline \multicolumn{11}{|l|}{ Continued } \\
\hline
\end{tabular}




\begin{tabular}{|c|c|c|c|c|c|c|c|c|c|c|}
\hline Study & Study design & Location & $\begin{array}{l}\text { Endocrine } \\
\text { therapy use }\end{array}$ & Sample size & $\begin{array}{l}\text { Sample age } \\
\text { (range, mean) }\end{array}$ & $\begin{array}{l}\text { Number } \\
\text { of tests in } \\
\text { battery }\end{array}$ & $\begin{array}{l}\text { Methodological } \\
\text { quality }\end{array}$ & $\begin{array}{l}\text { Definition } \\
\text { of cognitive } \\
\text { decline }\end{array}$ & $\begin{array}{l}\text { Time point(s) } \\
\neq\end{array}$ & $\begin{array}{l}\text { Prevalence } \\
\% \text { (95\% } \\
\text { CIs where } \\
\text { available) }\end{array}$ \\
\hline $\begin{array}{l}\text { Kreukels } \\
2008^{74}\end{array}$ & Cohort & Netherlands & Yes & 63 & 46.6 & 10 & High & $\begin{array}{l}\text { Scores lower } \\
\text { than } 2 \text { SD on } \\
\text { at least three } \\
\text { tests }\end{array}$ & $\mathrm{T} 3 / \mathrm{T} 4$ & 33 \\
\hline Lange $2016^{15}$ & Cohort & France & Yes & 58 & $65-81,70$ & 8 & High & $\begin{array}{l}\text { A significant } \\
\text { change in a } \\
\text { domain score }\end{array}$ & $\mathrm{T} 2$ & 64 \\
\hline $\begin{array}{l}\text { Mehlsen } \\
2009^{75}\end{array}$ & $\begin{array}{l}\text { Cross-sec- } \\
\text { tional }\end{array}$ & Denmark & Yes & 36 & 48.6 & 13 & High & $\begin{array}{l}\text { Decline on } \\
\text { at least } 3 \text { of } \\
\text { the cognitive } \\
\text { measures }\end{array}$ & $\mathrm{T} 2$ & 29 \\
\hline $\begin{array}{l}\text { Mehnert } \\
2007^{76}\end{array}$ & RCT & Germany & Yes & 23 & $33-65,53$ & 18 & Moderate & $\begin{array}{l}\text { Scores lower } \\
\text { than } 1.4 \mathrm{SD} \\
\text { on at least } \\
\text { four tests }\end{array}$ & T6 & 13 \\
\hline $\begin{array}{l}\text { Menning } \\
2016^{77}\end{array}$ & Cohort & Netherlands & Yes & 31 & 49.8 & 11 & High & $\begin{array}{l}\text { Multivariate } \\
\text { normative } \\
\text { comparison } \\
\text { compar- } \\
\text { ing against } \\
\text { distribution } \\
\text { of scores in } \\
\text { control group }\end{array}$ & $\mathrm{T} 3$ & 16 \\
\hline $\begin{array}{l}\text { Reid-Arndt } \\
2009^{78}\end{array}$ & $\begin{array}{l}\text { Cross sec- } \\
\text { tional study }\end{array}$ & United States & Yes & 46 & 53.4 & 11 & High & $\begin{array}{l}\text { Scores of } \\
\text { below } 1 \text { SD }\end{array}$ & $\mathrm{T} 2$ & $\begin{array}{l}\text { Executive } \\
\text { function- } \\
\text { ing: } 22 \\
\text { Verbal fluency: } \\
41\end{array}$ \\
\hline $\begin{array}{l}\text { Reid-Arndt } \\
2010^{79}\end{array}$ & $\begin{array}{l}\text { Prospective } \\
\text { longitudinal }\end{array}$ & United States & Yes & 39 & 53.4 & 7 & High & $\begin{array}{l}\text { Below } 1 \mathrm{SD} \text { as } \\
\text { indicative of } \\
\text { mild impair- } \\
\text { ment and } \\
\text { below } 1.5 \mathrm{SD} \\
\text { for moderate } \\
\text { impairment }\end{array}$ & $\mathrm{T} 3, \mathrm{~T} 4$ & $\begin{array}{l}\text { WMS Log } \\
\text { Mem: } \\
\text { T3: } 6 \\
\text { T4: } 33 \\
\text { RAVLT } \\
\text { T3:13 } \\
\text { T4: } 6 \\
\text { WMS Log } \\
\text { Mem II T3: } 3 \\
\text { T4: } 33 \\
\text { Trails A } \\
\text { T3: } 3 \\
\text { T4: } 9 \\
\text { Trails B } \\
\text { T3: 15 } \\
\text { T4: } 24 \\
\text { SCW } \\
\text { T3: } 3 \\
\text { T4: } 3 \\
\text { COWA } \\
\text { T3: } 0 \\
\text { T4: } 9 \\
\text { Category flu- } \\
\text { ency: T3: } 0 \\
\text { T4: } 16\end{array}$ \\
\hline Ruzich $2007^{80}$ & $\begin{array}{l}\text { Prospective } \\
\text { longitudinal }\end{array}$ & Australia & Yes & 35 & $30-66,53$ & 15 & Moderate & $\begin{array}{l}\text { Scores below } 1 \\
\text { SD on at least } \\
\text { two tests }\end{array}$ & $\mathrm{T} 1, \mathrm{~T} 2, \mathrm{~T} 3$ & $\begin{array}{l}\text { T1: } 15 \\
\text { T2: } 37 \\
\text { T3: } 30\end{array}$ \\
\hline $\begin{array}{l}\text { Schagen } \\
1999^{53}\end{array}$ & Cohort & Netherlands & Yes & 39 & 47.1 & 14 & Moderate & $\begin{array}{l}\text { Scores below } 2 \\
\text { SD on at least } \\
\text { three tests }\end{array}$ & T5 & 28 \\
\hline $\begin{array}{l}\text { Schagen } \\
2002^{81}\end{array}$ & $\begin{array}{l}\text { Cross-sec- } \\
\text { tional }\end{array}$ & Netherlands & Yes & $\begin{array}{l}\text { Two groups } \\
\text { of different } \\
\text { chemotherapy } \\
\text { regimes: } \\
\text { FECF: } 23 \\
\text { CMF }: 31\end{array}$ & 50.3 & 13 & High & $\begin{array}{l}\text { Number of } \\
\text { tests with } \\
\text { scores below } \\
2 \text { SD }\end{array}$ & T5 & $\begin{array}{l}\text { FEC: } 9 \\
\text { CMF: } 13\end{array}$ \\
\hline $\begin{array}{l}\text { Schagen } \\
2006^{82}\end{array}$ & RCT & Netherlands & Yes & 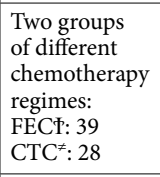 & 45 & $\begin{array}{l}10 \text { tests (not } \\
\text { listed) }\end{array}$ & High & $\begin{array}{l}\text { Scores below } 2 \\
\text { SD on at least } \\
\text { three tests }\end{array}$ & $\mathrm{T} 3$ & $\begin{array}{l}\text { FEC: } 10 \\
\text { CTC: } 20\end{array}$ \\
\hline $\begin{array}{l}\text { Schrauwen } \\
2020^{41}\end{array}$ & Cohort & Belgium & No & 66 & $27-64,46.7$ & 5 & High & $\begin{array}{l}\text { Standardized } \\
\text { difference } \\
\text { score exceed- } \\
\text { ing }-2.5 \mathrm{SD} \\
\text { on at least one } \\
\text { test }\end{array}$ & T2 & 24 \\
\hline
\end{tabular}




\begin{tabular}{|c|c|c|c|c|c|c|c|c|c|c|}
\hline Study & Study design & Location & $\begin{array}{l}\text { Endocrine } \\
\text { therapy use }\end{array}$ & Sample size & $\begin{array}{l}\text { Sample age } \\
\text { (range, mean) }\end{array}$ & $\begin{array}{l}\text { Number } \\
\text { of tests in } \\
\text { battery }\end{array}$ & $\begin{array}{l}\text { Methodological } \\
\text { quality }\end{array}$ & $\begin{array}{l}\text { Definition } \\
\text { of cognitive } \\
\text { decline }\end{array}$ & $\underset{\neq}{\operatorname{Time}}$ & $\begin{array}{l}\text { Prevalence } \\
\%(95 \% \\
\text { CIs where } \\
\text { available) }\end{array}$ \\
\hline Shilling $2005^{83}$ & Cohort & UK & Yes & 50 & 51.1 & 8 & High & \begin{tabular}{|l|} 
Reliable \\
decline on two \\
or more tests
\end{tabular} & $\mathrm{T} 2$ & 34 \\
\hline Stewart $2008^{84}$ & Cohort & Canada & Yes & 61 & $50-66,57.5$ & 18 & High & \begin{tabular}{|l|} 
Two or more \\
SRB scores of \\
-2.0 or less \\
on at least two \\
tests
\end{tabular} & $\mathrm{T} 2$ & 31 \\
\hline $\begin{array}{l}\text { Stouten- } \\
\text { Kemperman } \\
2015^{85}\end{array}$ & RCT & Netherlands & Yes & $\begin{array}{l}\text { Two groups } \\
\text { of different } \\
\text { chemotherapy } \\
\text { regimes: } \\
\text { Conventional } \\
\text { dose }=24 \\
\text { High dose }=17\end{array}$ & $56.3-59.8$ & 8 & Moderate & \begin{tabular}{|l|} 
Score larger \\
than 2SDs \\
below the \\
mean consid- \\
ered impaired \\
on a test. The \\
fifth percentile \\
of the overall \\
impair- \\
ment score \\
of healthy \\
control scores \\
was cutoff for \\
impairment \\
\end{tabular} & T7 & $\begin{array}{l}\text { Conventional } \\
\text { dose: } 8 \\
\text { High dose: } 11\end{array}$ \\
\hline Syarif $2019^{44}$ & $\begin{array}{l}\text { Cross-sec- } \\
\text { tional }\end{array}$ & Indonesia & Yes & 82 & 43 & 1 & Low & Not reported & Unclear & \begin{tabular}{|l|}
87 (mild- \\
serious \\
impairment)
\end{tabular} \\
\hline $\begin{array}{l}\text { Van Dam } \\
1998^{42}\end{array}$ & RCT & Netherlands & Yes & $\begin{array}{l}\text { FEC }: 36 \\
\text { CTC }^{\neq}: 34\end{array}$ & $\begin{array}{l}\text { FEC: } 48.1 \\
\text { CTC: } 45.5\end{array}$ & 13 & High & $\begin{array}{l}\text { Scores below 2 } \\
\text { SD on at least } \\
\text { three tests }\end{array}$ & T5 & $\begin{array}{l}\text { FEC: } 17 \\
\text { CTC: } 32\end{array}$ \\
\hline $\begin{array}{l}\text { Van Dyk } \\
2018^{86}\end{array}$ & $\begin{array}{l}\text { Cross-sec- } \\
\text { tional }\end{array}$ & United States & No & 20 & 47 & 17 & Moderate & \begin{tabular}{|l|} 
Impaired \\
by ICCTF \\
guidelines
\end{tabular} & $\mathrm{T} 2$ & 45 \\
\hline $\begin{array}{l}\text { Vearncombe } \\
2009^{87}\end{array}$ & $\begin{array}{l}\text { Prospective } \\
\text { longitudinal }\end{array}$ & Australia & No & 136 & 49.38 & 10 & High & $\begin{array}{l}\text { Reliable } \\
\text { change on } \\
\text { at least } 2 \\
\text { measures } \\
\end{array}$ & $\mathrm{T} 2$ & 17 \\
\hline Wefel $2010^{88}$ & $\begin{array}{l}\text { Prospective } \\
\text { longitudinal }\end{array}$ & United States & Yes & 42 & $33-65,48,4$ & 6 & High & \begin{tabular}{|l|} 
Scores below \\
2SD on one \\
test
\end{tabular} & $\mathrm{T} 2, \mathrm{~T} 4$ & $\begin{array}{l}\text { T2:65 } \\
\text { T4:61 }\end{array}$ \\
\hline $\begin{array}{l}\text { Wieneke } \\
1995^{89}\end{array}$ & $\begin{array}{l}\text { Cross sec- } \\
\text { tional }\end{array}$ & United States & Yes & 28 & $28-54,42$ & 15 & Moderate & $\begin{array}{l}\text { Score below } \\
2 \text { SD on at } \\
\text { least one test } \\
\text { classified as } \\
\text { moderate } \\
\text { impairment }\end{array}$ & $\begin{array}{l}\text { T2-T4 (varies } \\
\text { across period) }\end{array}$ & 75 \\
\hline
\end{tabular}

Table 3. Summary of included studies employing objective neuropsychological testing to assess cognitive impairment. $₹ \mathrm{~T} 1$ = During chemotherapy treatment, $\mathrm{T} 2=$ Just after cessation of treatment, $\mathrm{T} 3=6$ months after treatment cessation, T4 $=1$ year after treatment cessation, T5 $=2-3$ years after treatment cessation, T6 $=5$ 10 years after treatment cessation, $\mathrm{T} 7=\geq 10$ years after treatment cessation. ${ }^{\star}$ Standardised regression based. TFluorouracil, epirubicin, cyclophosphamide. ${ }^{\alpha}$ Cyclophosphamide, methotrexate, 5 -fluorouracil. ${ }^{\neq}$Four cycles of FEC followed by cyclophosphamide, thiotepa, and carboplatin.

\section{Meta-analysis}

Pooled prevalence. It was considered that meta-analysis was appropriate for further investigation of prevalence estimates derived from studies utilizing objective neuropsychological tests. Thirty-seven prevalence estimates from 27 articles were included in the meta-analysis, grouped based on time point of prevalence measure (from $\mathrm{T} 1$ to T5). Only one study contributed data at each of $\mathrm{T} 6^{76}$, and $\mathrm{T} 7^{85}$ and consequently these time points and studies were not included. Three studies were excluded from entry since prevalence was reported based on cognitive domain or test type $\mathrm{p}^{72,78,79}$. A further three studies were excluded since the timing of measure was unclear $^{44}$, or was performed across multiple time points as defined for this review ${ }^{74,89}$. In two studies, prevalence estimates were reported based on chemotherapy regime ${ }^{81,82}$. In order to ensure comparability with the other included studies only estimates for the standard dose chemotherapy regime from Schagen et al. $2006^{82}$ were included in the meta-analysis. Prevalence estimates reported by chemotherapy regimen in Schagen et al. $2002^{81}$ were pooled.

The overall random-effects pooled prevalence (95\% CIs) of cognitive impairment from time points T1-T5 respectively was 34\% (24-44), 30\% (20-40), 23\% (16-31), 31\% (14-50\%), and 21\% (15-28) (Fig. 5). Figure 6 synthesises these findings narratively across the time-points with the inclusion of the single individual study estimate contributed from ${ }^{76}$ and $^{85}$ at T6 and T7. There is a general downward trend in prevalence across time, although substantial imprecision in the effect at T4 with a slightly increased prevalence than might be expected if observing the trend. 


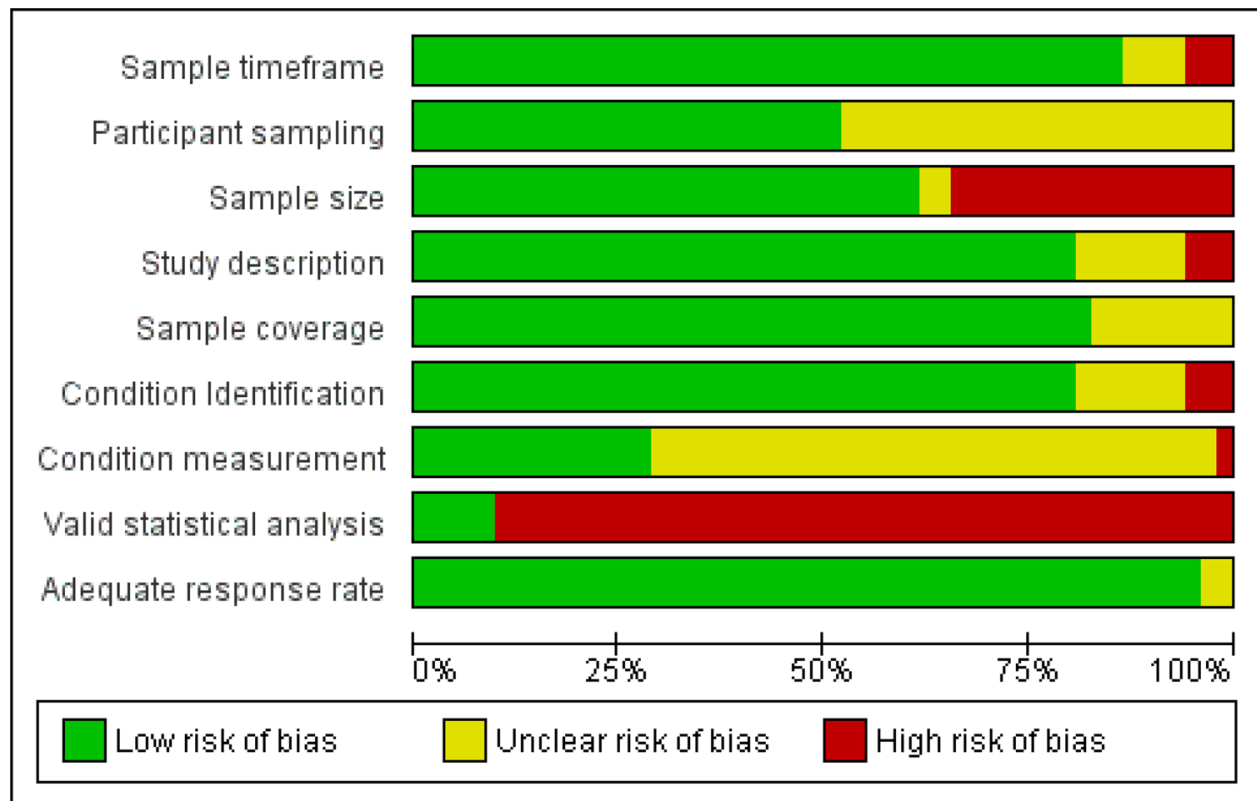

Figure 2. Risk of bias graph: review authors' judgements about each methodological quality item presented as percentages across all included studies. Study number $=52$.

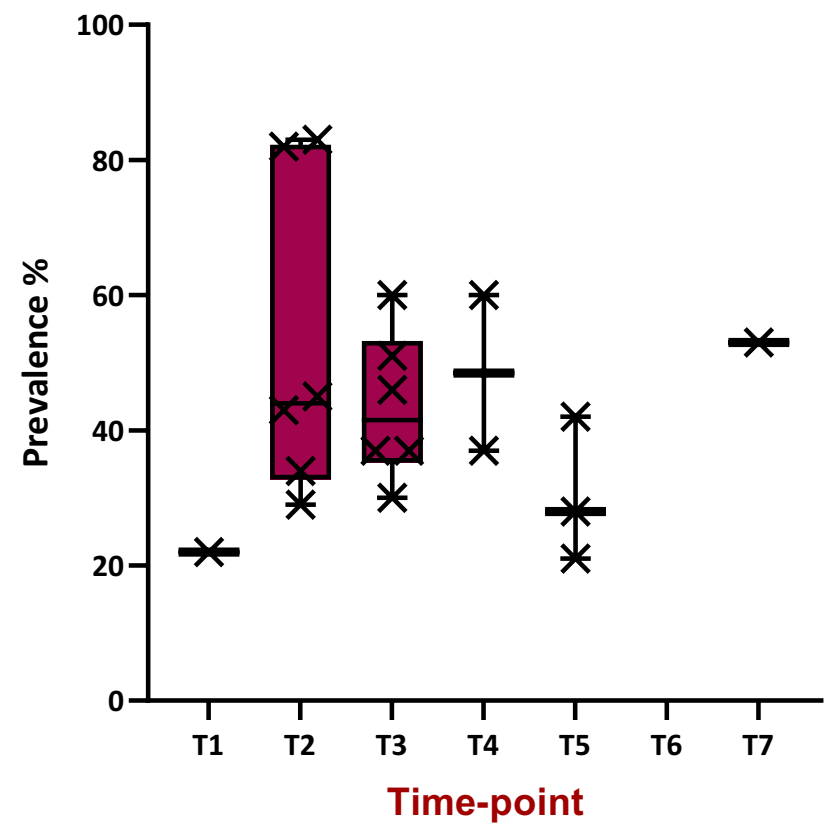

Figure 3. Boxplot of prevalence reported for cognitive impairment, via self-report methods, across the timepoints included in the review (data from 12 studies in Tables 1, 3 studies were excluded due to mixed or unclear timing of assessment $\left.{ }^{54,55,58}\right)$.

Heterogeneity and publication bias. Heterogeneity as determined by the $I^{2}$ statistic was universally high with the exception of $\mathrm{T} 5$ (where there were limited estimates) at 73\%, 90\%, 64\%, 89\% and 0 (T1-T5 respectively). Sensitivity analyses performed included removal of each study in turn, analysing untransformed data, considering the effect of the quality score and using a fixed effects model. In general, results were similar after these analyses (supplementary material S8). However, removal of Jansen et al. $2011^{70}$ at T1 had a significant effect reducing $I^{2}$ by $10 \%$ to $63 \%$ (potentially as a result of the impairment criteria used as described below). It is noteworthy that removal of the Andrysak 2018 study $^{64}$, which only employed one cognitive test, did not impact the heterogeneity estimates, nor did removal of the studies with cross-sectional designs ${ }^{75,86}$. 


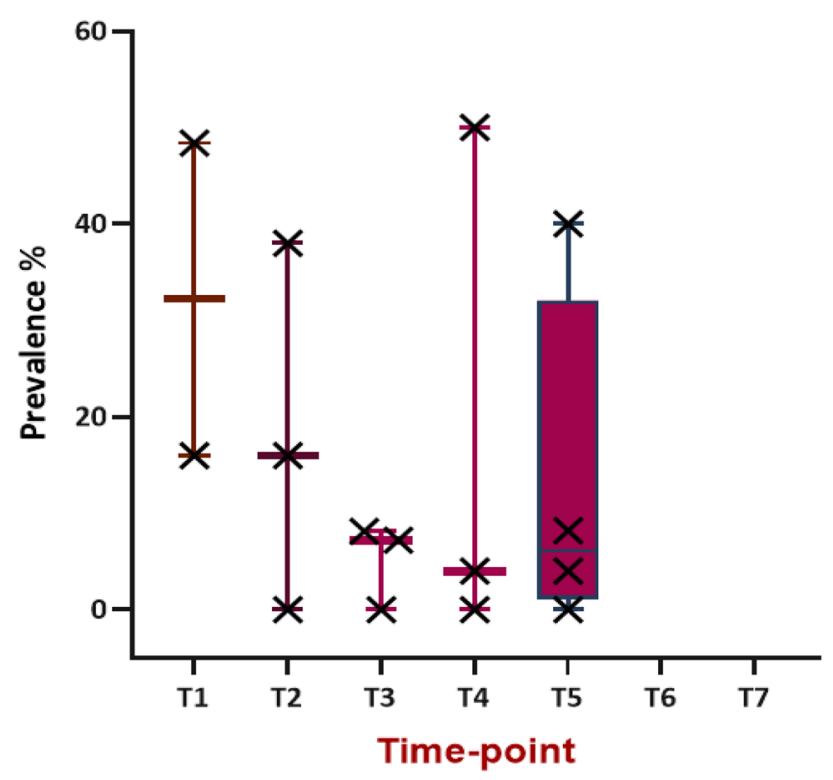

Figure 4. Boxplot of prevalence reported for cognitive impairment, via short cognitive screening methods, across the time-points included in the review. $\mathrm{Ng}$ et al. $2018^{52}$ was excluded from the calculation since a range was reported, and only considering moderate-severe classification of impairment from ${ }^{26} \mathrm{and}^{25}$.

In order to explain the heterogeneity in the pooled effects sizes the influence of stringency of criteria for diagnosing cognitive impairment was explored via subgroup analysis. Only prevalence reports from T2 and T3 were subjected to subgroup analyses based on the number of studies contributing data at these time-points, and the number in each analysis when split into sub-groups. At these time-points, four studies were considered to have used less stringent test methods ${ }^{70,75,80,88}$. Heterogeneity remained high after sub-group analysis with the high stringency sub-group having a pooled prevalence of $25 \%$ (95\% CIs: $16-35), I^{2}=90 \%$ at T2, and $23 \%(95 \%$ CIs: $13-34$ ), $I^{2}=74 \%$ at T3 (Fig. 7 ).

Meta-regression was performed in an attempt to account for the remaining heterogeneity. The results of five individual meta-regression analyses performed at T2 based on (1) the continuous variables of age, methodological quality and sample size, and (2) categorical variables of cognitive impairment criteria stringency and geographical location (3) subsets; Europe (reference group), North America, Asia-Pacific) are included in Table 4. Cognitive impairment stringency explained some of the variance in prevalence, accounting for $16 \%$ of the heterogeneity at T2 $(P=0.04)$. Sample size also accounted for $16 \%$ of the heterogeneity $(P=0.04)$ with increased sample size tending to result in a lower prevalence estimate. The remainder of the covariates could not account for the variance observed.

Meta-regression was then used to examine the relationship between sample size, cognitive impairment test stringency, and effect size. A test of this model yielded a Q-value of 6.99 with 2 degrees of freedom and corresponding $\mathrm{p}$-value of 0.03 . The test for heterogeneity yields a Q-value of 74.7 and a corresponding $\mathrm{p}$-value of 0 , implying that the variation of observed effects about the regression line falls within the range that cannot be explained by sampling error alone. The model is able to explain some $23 \%$ of the variance in true effects.

The doi plots for publication bias showed some asymmetry implying the presence of bias. The LF asymmetry index confirmed there was minor asymmetry at $\mathrm{T} 2$, and $\mathrm{T} 5$ and major asymmetry at $\mathrm{T} 4$. There was no evidence of publication bias at T1 and T3 (see supplementary material S9).

\section{GRADE certainty assessment and results}

The evidence presented across all the studies grouped by cognitive assessment methods was assessed using the GRADE (Grading of Recommendations Assessment, Development and Evaluation) approach (GRADEpro GDT: GRADEpro Guideline Development Tool [Software]). Results are presented in the Summary of Findings Table (Table 5). The certainty of evidence was graded as very low for all three-assessment types. Inconsistency was rated as serious for all groups of studies based on the wide variation in estimates and high calculated heterogeneity (neuropsychological testing). Imprecision was similarly rated as serious for all outcomes as a result of the wide confidence intervals. Publication bias was strongly suspected for the study group that used neuropsychological tests based on the doi plots and LFK index. Due to the method of synthesis used for the selfreport and short cognitive screening tool groups, publication bias was not able to be assessed and was therefore rated as undetected. 


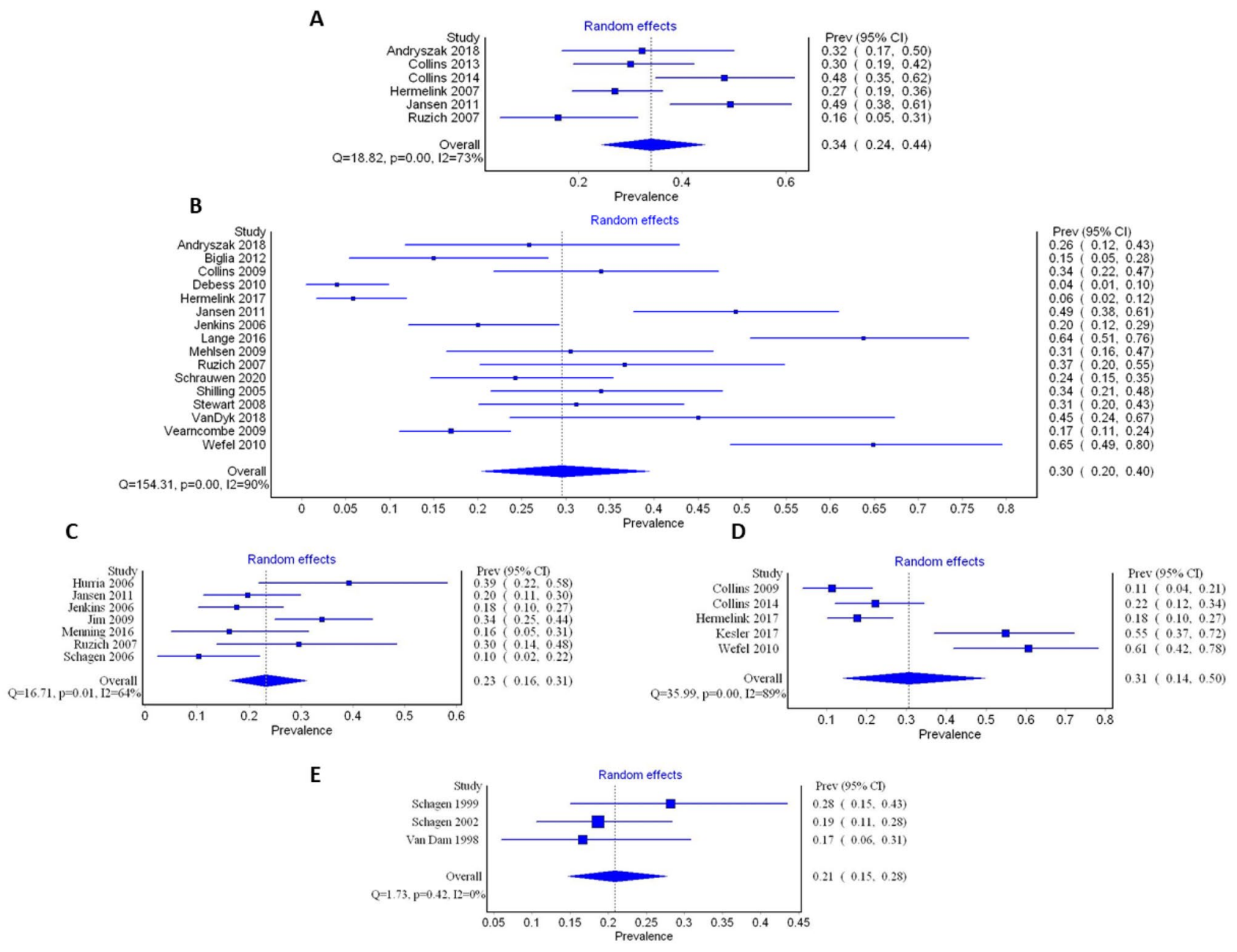

Figure 5. Forest plots of prevalence reported in studies utilising neuropsychological tests to diagnose cognitive impairment following chemotherapy treatment for breast cancer. (A) Cognitive assessment undertaken during chemotherapy treatment (T1), (B) Cognitive assessment undertaken just after cessation of chemotherapy treatment (T2), (C) Cognitive assessment undertaken 6 months after cessation of chemotherapy treatment (T3), (D) Cognitive assessment undertaken 1 year after cessation of chemotherapy treatment (T4), (E) Cognitive assessment undertaken 2-3 years after cessation of chemotherapy treatment (T5).

\section{Discussion}

This is the first systematic review on prevalence of CICI after treatment for breast cancer to have included and compared the three commonly used assessment modalities of self-report, short cognitive screen and objective neuropsychological tests. In contrast to the only other published systematic review of prevalence in CICI in this patient population ${ }^{2}$, meta-analytic techniques were applied to allow reporting of pooled effects, and studies which performed cognitive assessment beyond the breast cancer treatment period were also included. Prevalence estimates derived ranged from 0 to $83 \%$ across all time-points and all cognitive test methods. This crude figure is highly comparable with the commonly cited range of $12-82 \%$ based on the narrative review of Janelsins et al. $2014^{14}$. The current review allows greater examination of variability in these prevalence rates, through examination of the influence of factors such as test method, time since treatment and age on rates.

Impact of assessment tool choice on prevalence rates. There are striking differences in the average and maximum prevalence values reported based on method of cognitive assessment. Self-report of cognitive impairment yielded the highest values. There is substantially less variability between values obtained by short cognitive screen and neuropsychological test batteries. This difference in outcomes is to be expected for a number of reasons: (1) patient mood has been shown to have a relatively greater impact on subjective reports of impairment than actual cognitive performance. For example, aspects of mood such as anxiety, depression, poor quality of life and fatigue have all been shown to correlate with self-reported cognition complaints ${ }^{49,91-93} ;(2)$ expectations about treatment might influence self-report of cognitive symptoms. Study has shown that patients who were aware of the potential for cognitive side effects were more likely to report cognitive function issues than those who were unaware of the possibility ${ }^{94}$; (3) patients may have been functioning above the normal range of cognitive function prior to treatment, so whilst they experience a decline, their cognitive performance 


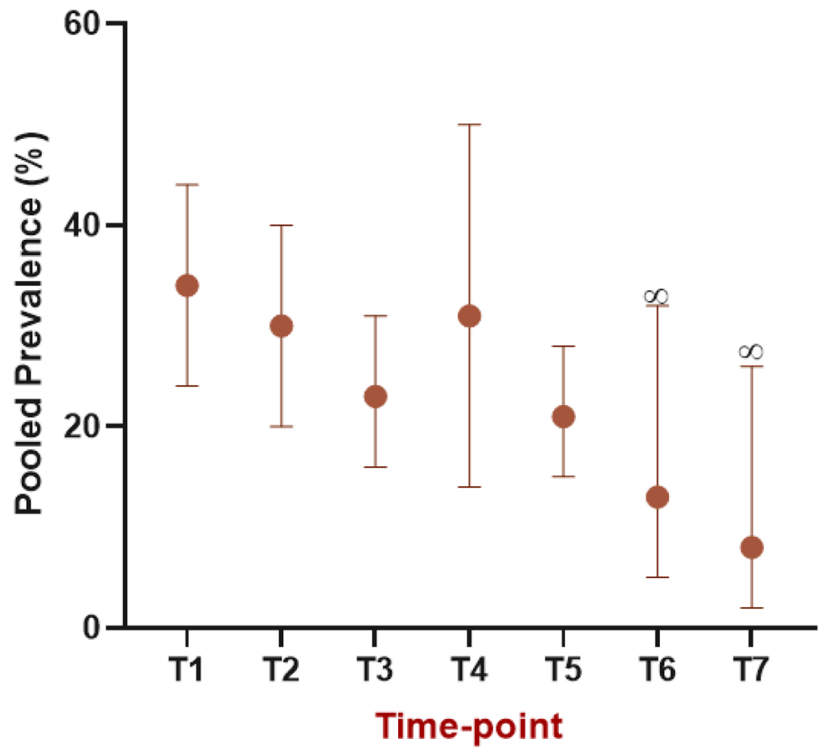

Figure 6. Prevalence reported for cognitive impairment, via neuropsychological test methods, across the timepoints included in the review. Data represent pooled prevalence estimates derived from meta-analysis, with $95 \%$ CIs. $\infty$-values are not pooled figures but represent individual values from one study.

falls within the normal range ${ }^{95,96}$; (4) the battery of objective tests used are ineffective at diagnosing subtle impairment ${ }^{95,96}$, this may especially be of concern with the use of short cognitive screening tools ${ }^{97}$; $(5)$ objective tests are ecologically invalid by failing to represent real-life situations where patients may experience the impairment ${ }^{95,96}$. Finally, based on the studies included in this review it could be argued that some of the assessment criteria are relatively crude, for example the endorsement of an item with no grading of response $\mathrm{e}^{11,58,59}$ would likely lead to substantially higher reporting of issues, which may not actually differ from normal agerelated memory loss experienced by healthy individuals ${ }^{11,58,59}$. In fact a cursory glance at the prevalence reports in these studies shows that reported prevalences are higher than the mean for the group. This perhaps highlights a future need to only use validated self-report instruments for cognitive assessment.

Our findings are in line with previous study where discrepancy between results of cognitive testing and clinical interview or self-report has been highlighted, with subjective perception of cognitive failure commonly not being able to be confirmed with objective tests ${ }^{8,98}$. Perceived impairment may actually be an indicator of psychological distress, brought about by anxiety, pain and depression, rather than a measure of cognitive functional capacity ${ }^{8}$. However, in clinical care patient perception matters. Addressing any of these individual factors through treatments or lifestyle change may bring about improvements in the others to provide an overall benefit. Disentangling the relative contribution of each of these elements is complex, and perhaps meaningless, in a clinical patient population ${ }^{98}$. Perhaps, there is a need to consider whether objective testing can be improved to reduce this discrepancy, for instance utilizing tests which cross cognitive domains and are therefore truer to real-life. Gamification may have a role in this regard. Alternatively, a hybrid model for diagnosing cognitive impairment may be superior, with the inclusion of objective tests and self-report in a standardized, validated protocol.

In spite of the reduced variability observed with objective tests, there was still significant statistical heterogeneity observed on meta-analysis. Approximately $16 \%$ of this heterogeneity could be explained by the use of less stringent test criteria for diagnosing impairment. Additionally, pooled prevalence was noticeably altered when subgroups based on test stringency were analysed. This finding provides strong evidence of the need for researchers to agree to, and use standardised impairment criteria, in order to make findings comparable, reproducible, and ultimately useful to practitioners. In fact, given that the International Cognition and Cancer Task Force advocated for such harmonisation in $2011^{99}$, it is surprising that this has not yet occurred, but may reflect individual researcher differences in experience with, and available access to certain tests.

Impact of time since treatment on prevalence rates. Prevalence rates ascertained by short screening methods or larger batteries of tests showed a general downward trend over time, with a peak rate occurring during chemotherapy treatment. This finding is supported by pre-clinical and imaging studies in CICI, which demonstrate that, over time, partial resolution of the brain structural and functional impairments occurs ${ }^{22,23}$. However, it does contrast with the trend in prevalence determined by Dijkshoorn et al. $2021^{2}$ which only included studies using objective tests, and found elevated (although perhaps not significantly so) prevalence rates at greater than one year after treatment, compared to just after treatment with chemotherapy. Notably however, actual prevalence values calculated in the current review for objective tests are remarkably similar to those in Dijkshoorn et al. $2021^{2}$. Rates of 25\%,14\% and $27 \%$ were reported in that review at time-points of just after treatment, up to 1 year following, and greater than 1 year after treatment respectively ${ }^{2}$. This corresponds with $30 \%, 23 \%$ and $31 \%$ at equivalent time points in the current review. This similarity in rates is in spite of the differ- 


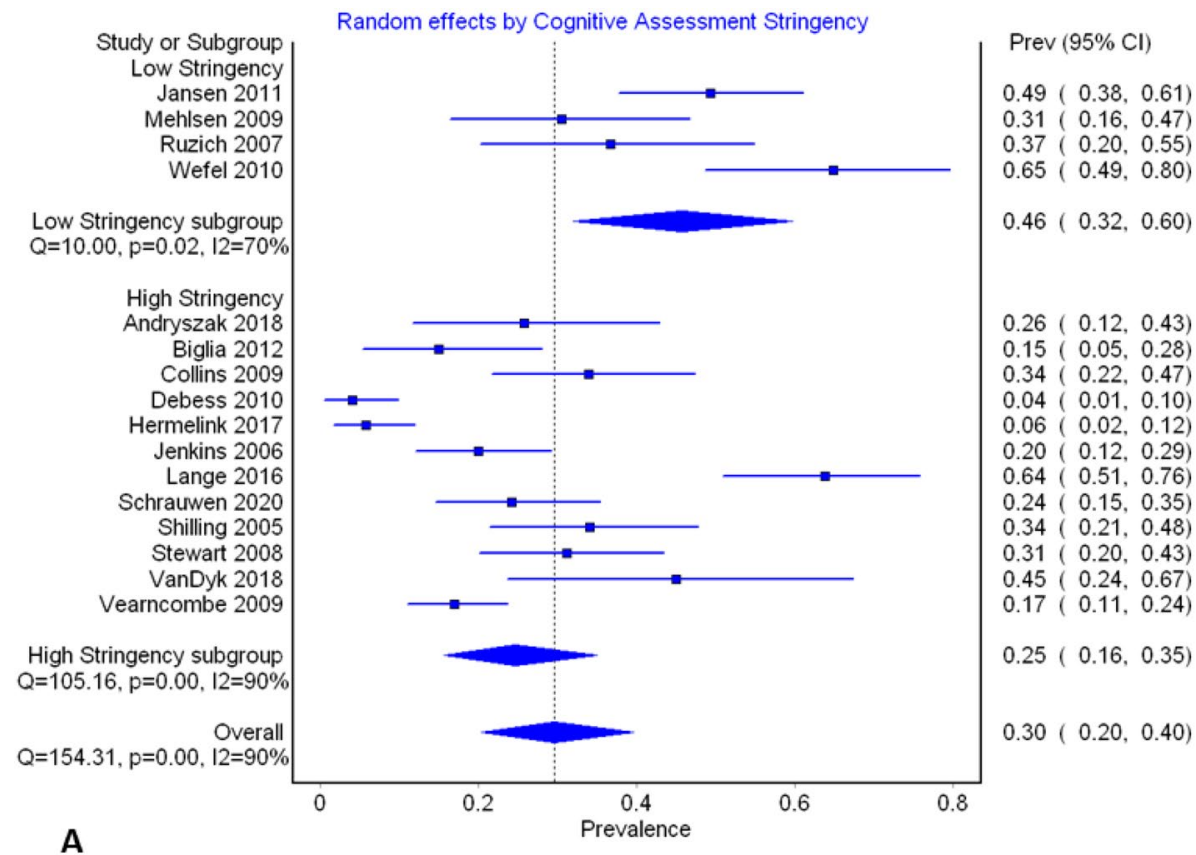

A

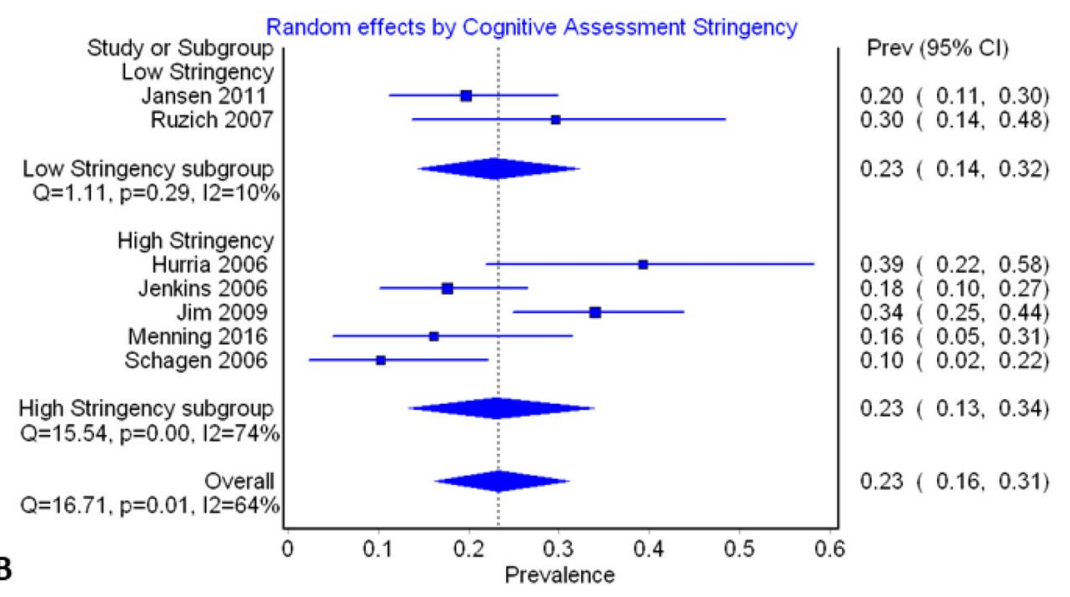

Figure 7. Forest plots of prevalence sub-grouped by stringency of cognitive assessment. (A) Cognitive assessment undertaken just after cessation of chemotherapy treatment (T2), (B) Cognitive assessment undertaken 6 months after cessation of chemotherapy treatment (T3).

\begin{tabular}{|c|c|c|c|}
\hline \multirow[b]{2}{*}{ T2- meta-regression } & \multicolumn{2}{|l|}{ Meta-regression } & \multirow[b]{2}{*}{$R^{2}$} \\
\hline & Mean difference (95\% CIs) & $P$ & \\
\hline Age & $0.06(-0.01$ to 0.13$)$ & 0.09 & 0.13 \\
\hline Quality Score & $-0.25(-4.14$ to 3.64$)$ & 0.9 & 0 \\
\hline Sample Size & $-0.02(-0.03$ to 0$)$ & 0.04 & 0.16 \\
\hline \multicolumn{4}{|l|}{ Location } \\
\hline \multicolumn{4}{|l|}{ Europe (ref group) } \\
\hline North America & $1.04(0.11-1.98)$ & \multirow{2}{*}{0.09} & \multirow{2}{*}{0.08} \\
\hline Asia-Pacific & $0.16(-1.14$ to 1.46$)$ & & \\
\hline \multicolumn{4}{|c|}{ Impairment criteria stringency } \\
\hline High stringency (ref group) & \multirow{2}{*}{$0.97(0.04-1.9)$} & \multirow{2}{*}{0.04} & \multirow{2}{*}{0.16} \\
\hline Low stringency & & & \\
\hline
\end{tabular}

Table 4. Results of four individual meta-regression analyses at $\mathrm{T} 2$ based on age, quality score, location and cognitive impairment criterion stringency. 


\begin{tabular}{|c|c|c|c|}
\hline Outcomes & $\begin{array}{l}\text { Ne of } \\
\text { participants } \\
\text { (studies) } \\
\text { Follow up }\end{array}$ & $\begin{array}{l}\text { Certainty of } \\
\text { the } \\
\text { evidence } \\
\text { (GRADE) }\end{array}$ & Impact \\
\hline $\begin{array}{l}\text { Prevalence of cognitive impairment diagnosed by self-report measures } \\
\text { (Self-report) }\end{array}$ & 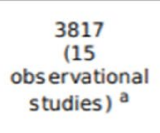 & $\underset{\text { VERY LOW }}{\oplus \text { b.c }}$ & $\begin{array}{l}\text { Prevalence rates for global impairment } \\
\text { varied from } 21-83 \% \text { (mean } 44 \% \text { ) across } \\
\text { time-points ranging from during } \\
\text { chemotherapy treatment to greater than } 10 \\
\text { years after cessation of treatment. }\end{array}$ \\
\hline $\begin{array}{l}\text { Prevalence of cognitive impairment diagnosed by short cognitive } \\
\text { screening tools (Short Cognitive Screening) }\end{array}$ & $\begin{array}{c}1533 \\
(9 \\
\text { observational } \\
\text { studies })\end{array}$ & $\underset{\text { VERY LOW }}{\oplus \text { b.c }}$ & $\begin{array}{l}\text { Prevalence rates for global impairment } \\
\text { varied from } 0-50 \% \text { (mean } 16 \% \text { ) across } \\
\text { time-points ranging from during } \\
\text { chemotherapy treatment to } 2-3 \text { years after } \\
\text { cessation of treatment. }\end{array}$ \\
\hline $\begin{array}{l}\text { Prevalence of cognitive impairment diagnosed by objective } \\
\text { neuropsychological tes ting (Objective Neuropsychological Test) }\end{array}$ & $\begin{array}{c}1938 \\
\text { (35 } \\
\text { observational }^{\text {studies })^{d}}\end{array}$ & $\underset{\substack{\text { VERY LOW } \\
\text { c.e.f }}}{\oplus O O O}$ & $\begin{array}{l}\text { The overall random-effects pooled } \\
\text { prevalence }(95 \% \text { Cls) of cognitive } \\
\text { impairment from time points which ranged } \\
\text { from during chemotherapy treatment, to } 2 \text { - } \\
3 \text { years after cessation of chemotherapy } \\
\text { (T1-T5) respectively was: } 34 \%(24-44), 30 \% \\
(20-40), 23 \% \text { ( } 16-31), 31 \%(14-50) \text {, and } 21 \% \\
(15-28) \text {. Late term prevalence at } 5-10 \\
\text { years, and greater than } 10 \text { years after } \\
\text { cessation of treatment, was } 13 \% \text { (5-32) and } \\
8 \% \text { ( } 2-26) \text { respectively (non-pooled data } \\
\text { with only one study contributing to each } \\
\text { value). }\end{array}$ \\
\hline
\end{tabular}

*The risk in the intervention group (and its $95 \%$ confidence interval) is based on the assumed risk in the comparison group and the relative effect of the intervention (and its $95 \% \mathrm{Cl}$ ).

Cl: Confidence interval

GRADE Working Group grades of evidence

High certainty: We are very confident that the true effect lies close to that of the es timate of the effect

Moderate certainty: We are moderately confident in the effect es timate: The true effect is likely to be close to the estimate of the effect, but there is a possibility that it is substantially different

is a possibility that it is substantially different

Very low certainty: We have very little confidence in the effect estimate: The true effect is likely to be substantially different from the estimate of effect

\section{Explanations}

a. 14 observational studies, 1 RCT

b. Point estimates varied widely across studies

c. Confidence intervals are wide

d. 30 observational studies, 5 RCT

e. Heterogeneity as determined by 12 univers ally high

f. Tests for publication bias show evidence of bias at a number of time-points

Table 5. GRADE Summary of Findings table for prevalence as determined through self-report, short cognitive screening tests and neuropsychological test batteries.

ences in methodology employed in the two reviews with the current review assimilating rates via meta-analytical techniques, and the previous review only including longitudinal studies such that impairment was based on a decline in cognitive function from baseline measures.

Conversely, the current review illustrates that prevalence rates as determined by self-report remain high (often above 40\%), and show little evidence of decline over time. The previously discussed suggestions explaining the general elevation in self-reported cognitive decline over measured impairment may also be at play here. Furthermore, at late follow-up stages patients will have likely returned to normal daily-life activities, such as work, and may have a greater perception of impairments as they return to navigating everyday tasks. This fits with current cancer rehabilitation evidence that suggests that survivors commonly suffer disabilities relating to activities of daily living such as doing housework and shopping ${ }^{100}$.

Whilst fewer studies have evaluated longer-term follow-up points, greater than 5 years following treatment cessation, there is evidence from both self-report and objective testing that some individuals are cognitively impaired at these time-points. This is an important finding for survivorship care and deserves dedicated research attention to fully elucidate impact.

Impact of age on prevalence rates. There is recognition of the potential for increased significance of chemobrain in older adults with cancer due to higher levels of pre-existing cognitive impairment in this age group $^{101}$. Furthermore, older adults may be more susceptible to cancer treatment toxicity leading to heightened symptoms ${ }^{15}$. Whilst it might therefore be predicted that CICI prevalence increases with age, the review did not find sufficient evidence to either support or refute this. Given there is wide variability in how people age even in the absence of medication effects studying this population group to come to reliable conclusions is likely problematic.

When meta-regression was applied, age in general was shown to have no effect on the high heterogeneity seen at T2. This lack of effect is unsurprising given that the studies included in the meta-regression had similar mean ages of the sample population, with relatively narrow ranges. Furthermore, for objective testing cognitive score results are usually compared against age-matched controls or some other age-based correction is applied, hence age is implicitly accounted for in experimental design. 
Only three studies specifically focused on patients over the age of $65^{15,45,46}$. Two of these studies ${ }^{15,45}$ used selfreport methods, with one reporting prevalence rates below the mean for the group ${ }^{45}$, and the other reporting rates high than the mean ${ }^{15}$. The third study ${ }^{46}$ utilized objective tests and reported a prevalence value higher than the reminder of the T3 subgroup. However, it also has the widest confidence intervals of the group suggesting the estimate to be imprecise. The study also used a low sample size, which may have influenced the estimate obtained. Noteworthy, is that the cohort study of Lange et al. 2016 also used cognitive tests, with diagnostic criteria categorized in this review as high stringency, and reported a high prevalence of $64 \%$ at T2. Moreover, confidence intervals were of similar width to other studies within the group ${ }^{15}$. This finding is perhaps the most suggestive of an effect of age on CICI prevalence and would be good grounds for dedicating future research effort to this question.

Strength of evidence on $\mathrm{CICl}$ prevalence. In order to account for any effect that low-quality studies might have had on the review findings, the meta-analyses incorporated a quality model, and a meta-regression using quality score as a variable was performed. These tools indicated that quality had minimal effect on prevalence rates or variances in the pooled estimates. As a result, there can be confidence that study quality has not affected review outcomes, at least for those studies that utilized objective cognitive assessment tools. Whilst, it is harder to make a formal determination of the impact of quality on the prevalence reports from studies using self-report and short cognitive screening tools, there is little to indicate that the results may have been unduly influenced by poor quality studies.

In all of the meta-analyses performed, with the exception of T5 where there were limited studies, moderate to high statistical heterogeneity was found. Sensitivity testing failed to account for any meaningful heterogeneity, and the subgroup analyses failed to reduce $I^{2}$ values substantially. Meta-regression did reveal an effect of cognitive impairment test stringency and sample size on variance. These factors accounted for $23 \%$ of the variance in effect seen at T2 in a combined model. The remainder of the heterogeneity could possibly be accounted for by methodological diversity due to the differing test batteries used, and further considerations relating to defining impairment levels that were not considered by the sub-group analysis. Additionally, clinical heterogeneity may have arisen as a result of treatments administered, patient menopausal status, or race.

There was evidence of publication bias at a number of time-points included in the meta-analysis, which lowers certainty in the findings from this analysis. However, there are a few considerations regarding assessment of publication bias in prevalence studies. Firstly, there is no specific guidance on assessment of publication bias when proportional data are involved. The issue being that funnel plots may be imprecise at the extremes of a proportion $^{102}$, and this may similarly apply to doi plots. It could be envisaged that the risk of publication bias should be lower in studies of prevalence since there is no favourable hypothesized outcome that may influence decision to publish. However, in the current review it is worth noting that in all of the studies the study objective was not solely around obtaining prevalence data on CICI. Taking this into consideration the possibility of publication bias likely exists, and was evidenced in this study.

Certainty of the evidence contributing to the findings of this review was assessed using the GRADE approach. It was determined that there was low certainty in the body of evidence contributing to the review. However, there is a lack of formal guidance from the GRADE working group on use of the methodology for reviews of prevalence, and the guidance criteria are not always applicable to these types of data. In spite of the lack of standardization, based on the assessment, conclusions should be made tentatively.

Study limitations. The review does have a number of limitations. First, due to the methodological diversity of the included studies, it was only possible to perform meta-analytic techniques on the studies that used neuropsychological tests. As a result, study weight has not been accounted for in the summary prevalence figures reported arising from self-report and short cognitive screening methods. This necessitates a need for extra circumspection around these values. Furthermore, even when meta-analytical techniques were used there was a resultant high statistical heterogeneity. Subgroup analyses and meta-regressions failed to account for most of this heterogeneity leaving the source(s) unknown, although some suggestions have been provided.

Second, due to the nature of the included studies with many having a repeated measures design it was not possible to use meta-analytical techniques to statistically compare prevalence across time-points; this being an aim of this review. Furthermore, in the current study using the All Time Points Meta-analysis approach assumption of independence between time-points was violated since some subjects contributed data at more than one time-point, whilst others only contribute at one time-point. This can lead to an ecological fallacy with a trend being seen on aggregate, that is not present at an individual level ${ }^{36}$. As a result there can only be low confidence in the direction of trends across time reported. Alternate approaches may be preferred when trends across time are of interest; for example, the use of a trend meta-analysis which uses regression modelling, or a change-in time meta-analysis where the primary study data is used to calculate differences between successive time-points or compared to baseline ${ }^{36}$. These alternate methods were not employed in this review since they require certain data to be provided in the primary studies such as a slope estimate for trend, or assume a longitudinal study design.

A few points regarding the conclusions that can be taken from this review are pertinent. The focus of the review was on estimating prevalence of CICI. However, as previously described cognition deficits may be caused by a range of factors associated with the cancer experience ${ }^{14}$. These may include the cancer itself and associated psychological distress and fatigue, or other administered treatments such as hormonal therapies. A further pertinent point is that study has suggested that race/ethnicity may influence prevalence of cognitive impairment, especially when self-report methods are used for assessment. ${ }^{56}$ This review did not specifically consider impact of ethnicity on rates of impairment reported. Moreover, whilst included studies commonly reported ethnicity data as part of the patient characteristics, results were rarely stratified by ethnic group. The impact of race on CICI 
prevalence therefore appears to be understudied, potentially leading to disparate health outcomes. It would be valuable in future evidence synthesis to consider this question specifically, allowing article selection to specifically address the influence of ethnicity. Furthermore, there is a clear need for researchers conducting primary research to consider, and address this issue in data collection and reporting. Additionally, studies eligible for inclusion in the review were not restricted to those with a longitudinal design, thus allowing for the contrast of findings with a baseline cognitive score. Based on these two points, it is impossible to say whether the cognitive deficits reported were wholly the result of chemotherapy, as opposed to a combination of cancer and multiple treatment-related effects. This concern is academic. From the perspective of patients and health-care providers it is the nature of cognitive decline that is important, not the origin of it. Since, these combinations of factors are inherent, and common to most women with breast cancer, it is considered that the review findings are generalizable.

\section{Conclusions}

This review is the first systematic comparison of prevalence rates for cognitive impairment in women with breast cancer that has considered all methods used to ascertain impairment, and evaluates long-term prevalence. Mean prevalence rates for CICI across all time-points were $44 \%$ using self-report and $6 \%$ using short cognitive screening. Pooled prevalence rates of between 21 and 34\% were derived for impairment diagnosed by objective neuropsychological tests. For all three assessment modalities the GRADE certainty in the evidence was rated as very low. Results therefore need to be interpreted with caution since the actual prevalence may be substantially different from this estimate.

Recommendations for practice. The findings of this review suggest that cognitive impairment may impact up to 1 in 3 patients at a level that is clinically significant. This impairment also extends beyond the treatment period and is often still apparent 2-3 years post treatment cessation. Health practitioners should consider the potential for cognitive impairment in breast cancer survivors, and guide them and their families towards sources of information and support. They may also like to discuss with patients some of the minimally invasive, non-therapeutic interventions that might ameliorate symptoms, for example cognitive training or physical activity $^{32}$.

Recommendations for research. The uncertainty around the true prevalence of cognitive impairment following cancer treatment leads to under-consideration of the condition, and consequently a lack of support being available for survivors. This leads to reduced patient quality of life through less meaningful engagement with work, family and daily activities, as well as economic impacts for both the individual and society. This review has highlighted a number of considerations for future research to provide greater certainty in the effect estimates. This includes increasing study sample sizes, utilising standardised cognitive test methods and batteries, and applying accepted, clinically relevant criteria for diagnosing impairment. With the increasing number of older cancer survivors, there is also a need for targeted research investigating this group. Furthermore, in order to tease apart the impact of relative impact of cancer alone, it would be valuable to perform further evidence synthesis to determine the prevalence of cognitive impairment in women with breast cancer, who have not received chemotherapy treatment. Acquiring accurate prevalence data will better inform survivorship strategies by allowing appropriate delivery of targeted resources and services, and informed resource allocation for this effort. Access to accurate prevalence estimates will also inform, and perhaps encourage, the development of evidence-based practice guidelines for CICI.

Received: 18 August 2021; Accepted: 17 January 2022

Published online: 08 February 2022

\section{References}

1. Nardin, S. et al. Breast cancer survivorship, quality of life, and late toxicities. Front. Oncol. https://doi.org/10.3389/fonc.2020. 00864 (2020).

2. Dijkshoorn, A. B. C. et al. Prevalence of cognitive impairment and change in patients with breast cancer: A systematic review of longitudinal studies. Psychooncology 30, 635-648. https://doi.org/10.1002/pon.5623 (2021).

3. Ahles, T. A., Root, J. C. \& Ryan, E. L. Cancer- and cancer treatment-associated cognitive change: An update on the state of the science. J. Clin. Oncol. 30(30), 3675-3686. https://doi.org/10.1200/JCO.2012.43.0116 (2012).

4. Mounier, N. M., Abdel-Maged, A. E., Wahdan, S. A., Gad, A. M. \& Azab, S. S. Chemotherapy-induced cognitive impairment (CICI): An overview of etiology and pathogenesis. Life Sci. 258, 118071. https://doi.org/10.1016/j.lfs.2020.118071 (2020).

5. Seigers, R., Schagen, S., Van Tellingen, O. \& Dietrich, J. Chemotherapy-related cognitive dysfunction: Current animal studies and future directions. Brain Imaging Behav. 7, 453-459 (2013).

6. George, R. P., Semendric, I., Hutchinson, M. R. \& Whittaker, A. L. Neuroimmune reactivity marker expression in rodent models of chemotherapy-induced cognitive impairment: A systematic scoping review. Brain Behav. Immun. https://doi.org/10.1016/j. bbi.2021.01.021 (2021).

7. Nguyen, L. D. \& Ehrlich, B. E. Cellular mechanisms and treatments for chemobrain: Insight from aging and neurodegenerative diseases. EMBO Mol. Med. 12, e12075. https://doi.org/10.15252/emmm.202012075 (2020).

8. Hutchinson, A. D., Hosking, J. R., Kichenadasse, G., Mattiske, J. K. \& Wilson, C. Objective and subjective cognitive impairment following chemotherapy for cancer: A systematic review. Cancer Treat. Rev. 38, 926-934 (2012).

9. Scherling, C. S. \& Smith, A. Opening up the window into "chemobrain": A neuroimaging review. Sensors (Basel) 13, 3169-3203. https://doi.org/10.3390/s130303169 (2013).

10. Wigmore, P. The effect of systemic chemotherapy on neurogenesis, plasticity and memory. Curr. Top. Behav. Neurosci. 15, 211-240. https://doi.org/10.1007/7854_2012_235(2013). 
11. Koppelmans, V. et al. Neuropsychological performance in survivors of breast cancer more than 20 years after adjuvant chemotherapy. J. Clin. Oncol. 30, 1080-1086. https://doi.org/10.1200/JCO.2011.37.0189 (2012).

12. Kanaskie, M. L. \& Loeb, S. J. The experience of cognitive change in women with breast cancer following chemotherapy. J. Cancer Surviv. 9, 375-387 (2015).

13. Mitchell, T. \& Turton, P. 'Chemobrain': concentration and memory effects in people receiving chemotherapy-A descriptive phenomenological study. Eur. J. Cancer Care 20, 539-548 (2011).

14. Janelsins, M. C., Kesler, S. R., Ahles, T. A. \& Morrow, G. R. Prevalence, mechanisms, and management of cancer-related cognitive impairment. Int. Rev. Psychiatry 26, 102-113. https://doi.org/10.3109/09540261.2013.864260 (2014)

15. Lange, M. et al. Decline in cognitive function in older adult swith early-stage breast cancer after adjuvant treatment. Oncologist 21, 1337-1348. https://doi.org/10.1634/theoncologist.2016-0014 (2016).

16. Greendale, G. A., Derby, C. A. \& Maki, P. M. Perimenopause and Cognition. Obstet. Gynecol. Clin. North Am. 38, 519-535. https://doi.org/10.1016/j.ogc.2011.05.007 (2011).

17. Vardy, J. Cognitive Function in Breast Cancer Survivors 387-419 (Springer, 2009).

18. Wefel, J. S., Lenzi, R., Theriault, R. L., Davis, R. N. \& Meyers, C. A. The cognitive sequelae of standard-dose adjuvant chemotherapy in women with breast carcinoma: Results of a prospective, randomized, longitudinal trial. Cancer 100, 2292-2299 (2004).

19. Lange, M. et al. Baseline cognitive functions among elderly patients with localised breast cancer. Eur. J. Cancer 50, 2181-2189 (2014).

20. Jansen, C. E., Dodd, M. J., Miaskowski, C. A., Dowling, G. A. \& Kramer, J. Preliminary results of a longitudinal study of changes in cognitive function in breast cancer patients undergoing chemotherapy with doxorubicin and cyclophosphamide. Psychooncology 17, 1189-1195 (2008).

21. Lange, M. \& Joly, F. How to identify and manage cognitive dysfunction after breast cancer treatment. J. Oncol. Pract. 13, 784-790. https://doi.org/10.1200/jop.2017.026286 (2017).

22. Inagaki, M. et al. Smaller regional volumes of brain gray and white matter demonstrated in breast cancer survivors exposed to adjuvant chemotherapy. Cancer 109, 146-156 (2007).

23. McDonald, B. C., Conroy, S. K., Ahles, T. A., West, J. D. \& Saykin, A. J. Gray matter reduction associated with systemic chemotherapy for breast cancer: A prospective MRI study. Breast Cancer Res. Treat. 123, 819-828 (2010).

24. Lv, L., Mao, S., Dong, H., Hu, P. \& Dong, R. Pathogenesis, assessments, and management of chemotherapy-related cognitive impairment (CRCI): An updated literature review. J. Oncol. 2020, 3942439. https://doi.org/10.1155/2020/3942439 (2020).

25. Brezden, C. B., Phillips, K. A., Abdolell, M., Bunston, T. \& Tannock, I. F. Cognitive function in breast cancer patients receiving adjuvant chemotherapy. J. Clin. Oncol. 18, 2695-2701. https://doi.org/10.1200/jco.2000.18.14.2695 (2000).

26. Fan, H. G. M. et al. Fatigue, menopausal symptoms, and cognitive function in women after adjuvant chemotherapy for breast cancer: 1- and 2-Year follow-up of a prospective controlled study. J. Clin. Oncol. 23, 8025-8032. https://doi.org/10.1200/JCO. 2005.01.6550 (2005).

27. Munn Z, M. S., Lisy K, Riitano D, Tufanaru C. Chapter 5: Systematic reviews of prevalence and incidence. In: JBI Manual for Evidence Synthesis (eds Aromataris, E., \& Munn, Z.) (JBI, 2020). Available from https://synthesismanual.jbi.global. https://doi. org/10.46658/JBIMES-20-06.

28. Page, M. J. et al. The PRISMA 2020 statement: An updated guideline for reporting systematic reviews. BMJ 372, n71. https:// doi.org/10.1136/bmj.n71 (2021).

29. Rodríguez Martín, B., Fernández Rodríguez, E. J., Rihuete Galve, M. I. \& Cruz Hernández, J. J. Study of chemotherapy-induced cognitive impairment in women with breast cancer. Int. J. Environ. Res. Public Health 17, 8896. https://doi.org/10.3390/ijerp h17238896 (2020).

30. Addington-Hall, J. \& McCarthy, M. Dying from cancer: Results of a national population-based investigation. Palliat Med 9, 295-305 (1995).

31. Gehring, K., Aaronson, N. K., Taphoorn, M. J. \& Sitskoorn, M. M. Interventions for cognitive deficits in patients with a brain tumor: An update. Expert Rev. Anticancer Ther. 10, 1779-1795 (2010).

32. Treanor, C. J. et al. Non-pharmacological interventions for cognitive impairment due to systemic cancer treatment. Cochrane Database Syst Rev. https://doi.org/10.1002/14651858.CD011325.pub2 (2016).

33. Craig, C., Monk, B., Farley, J. \& Chase, D. Cognitive impairment in gynecologic cancers: A systematic review of current approaches to diagnosis and treatment. Support Care Cancer 22, 279-287. https://doi.org/10.1007/s00520-013-2029-7 (2013).

34. Munn, Z., Moola, S., Lisy, K., Riitano, D. \& Tufanaru, C. Methodological guidance for systematic reviews of observational epidemiological studies reporting prevalence and cumulative incidence data. Int. J. Evid. Based Healthc. 13, 147-153. https:// doi.org/10.1097/xeb.0000000000000054 (2015).

35. Higgins. J. P. T. T. J., Chandler, J., Cumpston, M., Li, T., Page, M. J., Welch, V. A. (editors). Cochrane Handbook for Systematic Reviews of Interventions Version 6.2 (updated February 2021) (Cochrane, 2021). Available from www.training.cochrane.org/ handbook.

36. Peters, J. L. \& Mengersen, K. L. Meta-analysis of repeated measures study designs. J. Eval. Clin. Pract. 14, 941-950. https://doi. org/10.1111/j.1365-2753.2008.01010.x (2008).

37. Doi, S. A. \& Thalib, L. A quality-effects model for meta-analysis. Epidemiology 19, 94-100. https://doi.org/10.1097/EDE.0b013 e31815c24e7 (2008)

38. Barendregt, J., Doi, S., Lee, Y. Y., Pacella, R. \& Vos, T. Meta-analysis of prevalence. J. Epidemiol. Commun. Health 67, 974-978. https://doi.org/10.1136/jech-2013-203104 (2013).

39. Furuya-Kanamori, L., Barendregt, J. J. \& Doi, S. A. R. A new improved graphical and quantitative method for detecting bias in meta-analysis. Int. J. Evid. Based Healthc. 16, 195-203. https://doi.org/10.1097/xeb.0000000000000141 (2018).

40. Fontes, F., Pereira, S., Castro-Lopes, J. M. \& Lunet, N. A prospective study on the neurological complications of breast cancer and its treatment: Updated analysis three years after cancer diagnosis. Breast 29, 31-38. https://doi.org/10.1016/j.breast.2016. $06.013(2016)$

41. Schrauwen, W. et al. Heterogeneous response of chemotherapy-related cognitive decline in patients with breast cancer: A prospective study. J. Int. Neuropsychol. Soc. 26, 806-814. https://doi.org/10.1017/S1355617720000296 (2020).

42. van Dam, F. S. A. M. et al. Impairment of cognitive function in women receiving adjuvant treatment for high-risk breast cancer: High-dose versus standard-dose chemotherapy. J. Natl. Cancer Inst. 90, 210-218. https://doi.org/10.1093/jnci/90.3.210 (1998).

43. Pillai, U. S. et al. Late effects of breast cancer treatment and outcome after corrective interventions. Asian Pac. J. Cancer Prev. 20, 2673-2679. https://doi.org/10.31557/APJCP.2019.20.9.2673 (2019).

44. Syarif, H., Waluyo, A., Afiyanti, Y. \& Mansyur, M. Executive function in breast cancer survivors and the influencing factors. Enferm. Clin. 29(Suppl 2), 280-285. https://doi.org/10.1016/j.enfcli.2019.04.034 (2019).

45. Hurria, A. et al. Effect of adjuvant breast cancer chemotherapy on cognitive function from the older patient's perspective. Breast Cancer Res. Treat. 98, 343-348. https://doi.org/10.1007/s10549-006-9171-6 (2006).

46. Hurria, A. et al. Cognitive function of older patients receiving adjuvant chemotherapy for breast cancer: A pilot prospective longitudinal study. J. Am. Geriatr. Soc. 54, 925-931. https://doi.org/10.1111/j.1532-5415.2006.00732.x (2006).

47. Buwalda, B. \& Schagen, S. B. Is basic research providing answers if adjuvant anti-estrogen treatment of breast cancer can induce cognitive impairment?. Life Sci. 93, 581-588 (2013). 
48. Shilling, V., Jenkins, V., Fallowfield, L. \& Howell, T. The effects of hormone therapy on cognition in breast cancer. J. Steroid Biochem. Mol. Biol. 86, 405-412. https://doi.org/10.1016/j.jsbmb.2003.07.001 (2003).

49. Biglia, N. et al. Objective and self-reported cognitive dysfunction in breast cancer women treated with chemotherapy: A prospective study. Eur. J. Cancer Care 21, 485-492. https://doi.org/10.1111/j.1365-2354.2011.01320.x (2012).

50. Debess, J., Riis, J. Ø., Engebjerg, M. C. \& Ewertz, M. Cognitive function after adjuvant treatment for early breast cancer: A population-based longitudinal study. Breast Cancer Res. Treat. 121, 91-100. https://doi.org/10.1007/s10549-010-0756-8 (2010).

51. Jenkins, V. et al. A 3-year prospective study of the effects of adjuvant treatments on cognition in women with early stage breast cancer. Br. J. Cancer. 94, 828-834. https://doi.org/10.1038/sj.bjc.6603029 (2006).

52. Ng, T. et al. Distinct and heterogeneous trajectories of self-perceived cognitive impairment among Asian breast cancer survivors. Psychooncology 27, 1185-1192. https://doi.org/10.1002/pon.4635 (2018).

53. Schagen, S. B. et al. Cognitive deficits after postoperative adjuvant chemotherapy for breast carcinoma. Cancer 85, 640-650. https://doi.org/10.1002/(SICI) 1097-0142(19990201)85:3\%3c640::AID-CNCR14\%3e3.0.CO;2-G (1999).

54. Buchanan, N. D. et al. Post-treatment neurocognition and psychosocial care among breast cancer survivors. Am. J. Prev. Med. 49, S498-S508. https://doi.org/10.1016/j.amepre.2015.08.013 (2015).

55. Ganz, P. A. et al. Cognitive complaints after breast cancer treatments: Examining the relationship with neuropsychological test performance. J. Natl. Cancer Inst. 105, 791-801. https://doi.org/10.1093/jnci/djt073 (2013).

56. Janelsins, M. C. et al. Cognitive complaints in survivors of breast cancer after chemotherapy compared with age-matched controls: An analysis from a nationwide, multicentre, prospective longitudinal study. J. Clin. Oncol. 35, 506-514. https://doi.org/10.1200/ jco.2016.68.5826 (2017)

57. Rey, D. et al. Self-reported cognitive impairment after breast cancer treatment in young women from the ELIPPSE40 cohort: The long-term impact of chemotherapy. Breast J. 18, 406-414. https://doi.org/10.1111/j.1524-4741.2012.01275.x (2012).

58. Schmidt, J. E. et al. Prevalence of perceived cognitive dysfunction in survivors of a wide range of cancers: Results from the 2010 LIVESTRONG survey. J. Cancer Surviv. 10, 302-311. https://doi.org/10.1007/s11764-015-0476-5 (2016).

59. Shilling, V. \& Jenkins, V. Self-reported cognitive problems in women receiving adjuvant therapy for breast cancer. Eur. J. Oncol. Nurs. 11, 6-15. https://doi.org/10.1016/j.ejon.2006.02.005 (2007).

60. Tager, F. A. et al. The cognitive effects of chemotherapy in post-menopausal breast cancer patients: A controlled longitudinal study. Breast Cancer Res. Treat. 123, 25-34. https://doi.org/10.1007/s10549-009-0606-8 (2010).

61. Prokasheva, S., Faran, Y., Cwikel, J. \& Geffen, D. B. Analysis of memory deficits following chemotherapy in breast cancer survivors: Evidence from the doors and people test. J. Psychosoc. Oncol. 29, 499-514. https://doi.org/10.1080/07347332.2011.600751 (2011).

62. Ramalho, M., Fontes, F., Ruano, L., Pereira, S. \& Lunet, N. Cognitive impairment in the first year after breast cancer diagnosis: A prospective cohort study. Breast 32, 173-178. https://doi.org/10.1016/j.breast.2017.01.018 (2017).

63. Tchen, N. et al. Cognitive function, fatigue, and menopausal symptoms in women receiving adjuvant chemotherapy for breast cancer. J. Clin. Oncol. 21, 4175-4183. https://doi.org/10.1200/JCO.2003.01.119 (2003).

64. Andryszak, P., Wiłkość, M., Żurawski, B. \& Izdebski, P. Verbal memory in breast cancer patients treated with chemotherapy with doxorubicin and cyclophosphamide. Eur. J. Cancer Care 27(1), 1-11 (2018).

65. Collins, B., Mackenzie, J., Stewart, A., Bielajew, C. \& Verma, S. Cognitive effects of chemotherapy in post-menopausal breast cancer patients 1 year after treatment. Psychooncology 18, 134-143. https://doi.org/10.1002/pon.1379 (2009).

66. Collins, B., MacKenzie, J., Tasca, G. A., Scherling, C. \& Smith, A. Cognitive effects of chemotherapy in breast cancer patients: A dose-response study. Psychooncology 22, 1517-1527. https://doi.org/10.1002/pon.3163 (2013).

67. Collins, B., Mackenzie, J., Tasca, G. A., Scherling, C. \& Smith, A. Persistent cognitive changes in breast cancer patients 1 year following completion of chemotherapy. J. Int. Neuropsychol. Soc. 20, 370-379. https://doi.org/10.1017/s1355617713001215 (2014).

68. Hermelink, K. et al. Cognitive function during neoadjuvant chemotherapy for breast cancer: Results of a prospective, multicenter, longitudinal study. Cancer 109, 1905-1913. https://doi.org/10.1002/cncr.22610 (2007).

69. Hermelink, K. et al. Chemotherapy and Post-traumatic Stress in the Causation of Cognitive Dysfunction in Breast Cancer Patients. J Natl Cancer Inst https://doi.org/10.1093/jnci/djx057 (2017).

70. Jansen, C. E., Cooper, B. A., Dodd, M. J. \& Miaskowski, C. A. A prospective longitudinal study of chemotherapy-induced cognitive changes in breast cancer patients. Support Care Cancer 19, 1647-1656. https://doi.org/10.1007/s00520-010-0997-4 (2011).

71. Jim, H. S. L. et al. Cognitive functioning in breast cancer survivors: A controlled comparison. Cancer 115, 1776-1783. https:// doi.org/10.1002/cncr.24192 (2009).

72. Jung, M. S. \& Cimprich, B. Cognitive deficits in Korean women treated with chemotherapy for breast cancer. Cancer Nurs. 37, E31-E42. https://doi.org/10.1097/NCC.0b013e3182980383 (2014).

73. Kesler, S. R. et al. Predicting long-term cognitive outcome following breast cancer with pre-treatment resting state fMRI and random forest machine learning. Front. Hum. Neurosci. 11, 555. https://doi.org/10.3389/fnhum.2017.00555 (2017).

74. Kreukels, B. P. C., Van Dam, F. S. A. M., Ridderinkhof, K. R., Boogerd, W. \& Schagen, S. B. Persistent neurocognitive problems after adjuvant chemotherapy for breast cancer. Clin. Breast Cancer 8, 80-87. https://doi.org/10.3816/CBC.2008.n.006 (2008).

75. Mehlsen, M., Pedersen, A. D., Jensen, A. B. \& Zachariae, R. No indications of cognitive side-effects in a prospective study of breast cancer patients receiving adjuvant chemotherapy. Psychooncology 18, 248-257. https://doi.org/10.1002/pon.1398 (2009).

76. Mehnert, A. et al. The association between neuropsychological impairment, self-perceived cognitive deficits, fatigue and health related quality of life in breast cancer survivors following standard adjuvant versus high-dose chemotherapy. Patient Educ. Couns. 66, 108-118. https://doi.org/10.1016/j.pec.2006.11.005 (2007).

77. Menning, S. et al. Cognitive impairment in a subset of breast cancer patients after systemic therapy-results from a longitudinal study. J. Pain Symptom Manag. 52, 560-569.e561. https://doi.org/10.1016/j.jpainsymman.2016.04.012 (2016).

78. Reid-Arndt, S. A., Yee, A., Perry, M. C. \& Hsieh, C. Cognitive and psychological factors associated with early posttreatment functional outcomes in breast cancer survivors. J. Psychosoc. Oncol. 27, 415-434. https://doi.org/10.1080/07347330903183117 (2009).

79. Reid-Arndt, S. A., Hsieh, C. \& Perry, M. C. Neuropsychological functioning and quality of life during the first year after completing chemotherapy for breast cancer. Psychooncology 19, 535-544. https://doi.org/10.1002/pon.1581 (2010).

80. Ruzich, M., Ryan, B., Owen, C., Delahunty, A. \& Stuart-Harris, R. Prospective evaluation of cognitive function in patients with early breast cancer receiving adjuvant chemotherapy. Asia Pac. J. Clin. Oncol. 3, 125-133. https://doi.org/10.1111/j.1743-7563. 2007.00109.x (2007)

81. Schagen, S. B. et al. Late effects of adjuvant chemotherapy on cognitive function: A follow-up study in breast cancer patients. Ann. Oncol. 13, 1387-1397. https://doi.org/10.1093/annonc/mdf241 (2002).

82. Schagen, S. B., Muller, M. J., Boogerd, W., Mellenbergh, G. J. \& van Dam, F. S. A. M. Change in cognitive function after chemotherapy: A prospective longitudinal study in breast cancer patients. J. Natl. Cancer Inst. 98, 1742-1745. https://doi.org/10.1093/ jnci/djj470 (2006)

83. Shilling, V., Jenkins, V., Morris, R., Deutsch, G. \& Bloomfield, D. The effects of adjuvant chemotherapy on cognition in women with breast cancer-preliminary results of an observational longitudinal study. Breast 14, 142-150. https://doi.org/10.1016/j. breast.2004.10.004 (2005).

84. Stewart, A. et al. The cognitive effects of adjuvant chemotherapy in early stage breast cancer: A prospective study. Psychooncology 17, 122-130. https://doi.org/10.1002/pon.1210 (2008). 
85. Stouten-Kemperman, M. M. et al. Very late treatment-related alterations in brain function of breast cancer survivors. J. Int. Neuropsych. Soc. 21, 50-61. https://doi.org/10.1017/S1355617714001015 (2015).

86. Van Dyk, K., Bower, J. E., Crespi, C. M., Petersen, L. \& Ganz, P. A. Cognitive function following breast cancer treatment and associations with concurrent symptoms. npi Breast Cancer https://doi.org/10.1038/s41523-018-0076-4 (2018).

87. Vearncombe, K. J. et al. Predictors of cognitive decline after chemotherapy in breast cancer patients. J. Int. Neuropsych. Soc. 15, 951-962. https://doi.org/10.1017/S1355617709990567 (2009).

88. Wefel, J. S., Saleeba, A. K., Buzdar, A. U. \& Meyers, C. A. Acute and late onset cognitive dysfunction associated with chemotherapy in women with breast cancer. Cancer 116, 3348-3356. https://doi.org/10.1002/cncr.25098 (2010).

89. Wieneke, M. H. \& Dienst, E. R. Neuropsychological assessment of cognitive functioning following chemotherapy for breast cancer. Psychooncology 4, 61-66. https://doi.org/10.1002/pon.2960040108 (1995).

90. Jackson, D. \& Turner, R. Power analysis for random-effects meta-analysis. Res. Synth. Methods 8, 290-302. https://doi.org/10. 1002/jrsm.1240 (2017).

91. Castellon, S. A. et al. Neurocognitive performance in breast cancer survivors exposed to adjuvant chemotherapy and tamoxifen. J. Clin. Exp. Neuropsychol. 26, 955-969. https://doi.org/10.1080/13803390490510905 (2004).

92. Krolak, D., Collins, B., Weiss, L., Harris, C. \& Van der Jagt, R. Cognitive function and its relationship to other psychosocial factors in lymphoma survivors. Support Care Cancer 25, 905-913. https://doi.org/10.1007/s00520-016-3480-z (2017).

93. Boscher, C. et al. Perceived cognitive impairment in breast cancer survivors and its relationships with psychological factors. Cancers Basel 12, 3000. https://doi.org/10.3390/cancers12103000 (2020).

94. Schagen, S. B., Das, E. \& Vermeulen, I. Information about chemotherapy-associated cognitive problems contributes to cognitive problems in cancer patients. Psychooncology 21, 1132-1135. https://doi.org/10.1002/pon.2011 (2012).

95. Vardy, J. L. et al. Cognitive function in patients with colorectal cancer who do and do not receive chemotherapy: A prospective, longitudinal controlled study. J. Clin. Oncol. 33, 4085-4092. https://doi.org/10.1200/jco.2015.63.0905 (2015).

96. Bray, V. J., Dhillon, H. M. \& Vardy, J. L. Systematic review of self-reported cognitive function in cancer patients following chemotherapy treatment. J. Cancer Surviv. 12, 537-559. https://doi.org/10.1007/s11764-018-0692-x (2018).

97. Argyriou, A. A., Assimakopoulos, K., Iconomou, G., Giannakopoulou, F. \& Kalofonos, H. P. Either called “Chemobrain” or "Chemofog", the long-term chemotherapy-induced cognitive decline in cancer survivors is real. J. Pain Symptom Manage. 41, 126-139. https://doi.org/10.1016/j.jpainsymman.2010.04.021 (2011).

98. Bellens, A., Roelant, E., Sabbe, B., Peeters, M. \& van Dam, P. A. A video-game based cognitive training for breast cancer survivors with cognitive impairment: A prospective randomized pilot trial. Breast 53, 23-32. https://doi.org/10.1016/j.breast.2020.06.003 (2020).

99. Wefel, J. S., Vardy, J., Ahles, T. \& Schagen, S. B. International cognition and cancer task force recommendations to harmonise studies of cognitive function in patients with cancer. Lancet Oncol. 12(7), 703-708 (2011).

100. Neo, J., Fettes, L., Gao, W., Higginson, I. J. \& Maddocks, M. Disability in activities of daily living among adults with cancer: A systematic review and meta-analysis. Cancer Treat. Rev. 61, 94-106 (2017).

101. Loh, K. P. et al. Chemotherapy-related cognitive impairment in older patients with cancer. J. Geriatr. Oncol. 7, 270-280. https:// doi.org/10.1016/j.jgo.2016.04.008 (2016).

102. Hunter, J. P. et al. In meta-analyses of proportion studies, funnel plots were found to be an inaccurate method of assessing publication bias. J. Clin. Epidemiol. 67, 897-903. https://doi.org/10.1016/j.jclinepi.2014.03.003 (2014).

\section{Author contributions}

A.L.W conceptualised the project, carried out the searches, performed data selection and extraction and quality appraisal as well as writing the manuscript. R.P.G validated the work performed by A.L.W in data selection, extraction and quality appraisal. L.O'M was involved in development of the project and supervision. All of the authors reviewed and edited the manuscript.

\section{Funding}

This research did not receive any specific Grant from funding agencies in the public, commercial, or not-for-profit sectors. ALW was supported by a NHMRC Peter Doherty Biomedical Research Fellowship (APP1140072). RPG was supported by an Australian Government Research Training Program Scholarship.

\section{Competing interests}

The authors declare no competing interests.

\section{Additional information}

Supplementary Information The online version contains supplementary material available at https://doi.org/ 10.1038/s41598-022-05682-1.

Correspondence and requests for materials should be addressed to A.L.W.

Reprints and permissions information is available at www.nature.com/reprints.

Publisher's note Springer Nature remains neutral with regard to jurisdictional claims in published maps and institutional affiliations.

Open Access This article is licensed under a Creative Commons Attribution 4.0 International License, which permits use, sharing, adaptation, distribution and reproduction in any medium or format, as long as you give appropriate credit to the original author(s) and the source, provide a link to the Creative Commons licence, and indicate if changes were made. The images or other third party material in this article are included in the article's Creative Commons licence, unless indicated otherwise in a credit line to the material. If material is not included in the article's Creative Commons licence and your intended use is not permitted by statutory regulation or exceeds the permitted use, you will need to obtain permission directly from the copyright holder. To view a copy of this licence, visit http://creativecommons.org/licenses/by/4.0/.

(c) The Author(s) 2022 